\title{
Article \\ Innovative Analytical Method for X-Ray Imaging and Space-Resolved Spectroscopy of ECR Plasmas
}

\author{
Eugenia Naselli ${ }^{1 *} \mathbb{D}$, Richard Rácz ${ }^{2} \mathbb{D}$, Sandor Biri $^{2}$, Maria Mazzaglia $^{1} \mathbb{D}^{\mathbb{D}}$, Luigi Celona ${ }^{1}$, Santo Gammino $^{1}$, \\ Giuseppe Torrisi ${ }^{1}$, Zoltan Perduk ${ }^{2}$, Alessio Galatà ${ }^{3}$ and David Mascali ${ }^{1}$ (D) \\ 1 Istituto Nazionale di Fisica Nucleare, Laboratori Nazionali del Sud, Via S. Sofia 62, 95123 Catania, Italy; \\ eugenia.naselli@lns.infn.it; mazzaglia@lns.infn.it; celona@lns.infn.it; gammino@lns.infn.it; \\ peppetorrisi@lns.infn.it; davidmascali@lns.infn.it \\ 2 Institute for Nuclear Research (Atomki), Bem square 18/c, 4026 Debrecen, Hungary; rracz@atomki.mta.hu; \\ biri@atomki.hu; perduk@atomki.hu \\ 3 Istituto Nazionale di Fisica Nucleare, Laboratori Nazionali di Legnaro, Viale dell'Università 2, 35020 Legnaro, \\ Italy; alessio.galata@lnl.infn.it \\ * Correspondence: eugenia.naselli@lns.infn.it
}

\begin{abstract}
At INFN-LNS, and in collaboration with the ATOMKI laboratories, an innovative multidiagnostic system with advanced analytical methods has been designed and implemented. This is based on several detectors and techniques (Optical Emission Spectroscopy, RF systems, Interferopolarimetry, X-ray detectors) and here we focus on high resolution spatially-resolved X-ray spectroscopy, performed by means of a X-ray pin-hole camera setup operating in the $0.5-20 \mathrm{keV}$ energy domain. The diagnostic system was installed at a $14 \mathrm{GHz}$ Electron Cyclotron Resonance (ECR) ion source (ATOMKI, Debrecen), enabling high precision X-ray spectrally-resolved imaging of ECR plasmas heated by hundreds of Watts. The achieved spatial and energy resolution were $0.5 \mathrm{~mm}$ and $300 \mathrm{eV}$ at $8 \mathrm{keV}$, respectively. We here present the innovative analysis algorithm that we properly developed for obtaining Single Photon-Counted (SPhC) images providing the local plasma emitted spectrum in a High-Dynamic-Range (HDR) mode, by distinguishing fluorescence lines of the materials of the plasma chamber ( $\mathrm{Ti}, \mathrm{Ta}$ ) from plasma (Ar). This method allows a quantitative characterization of warm electrons population in the plasma (and its 2D distribution) which are the most important for ionization, and also to estimate local plasma density and spectral temperatures. The developed post-processing analysis is also able to remove the readout noise, that is often observable at very low exposure times (msec). The setup is now under update including fast shutters and trigger systems in order to allow simultaneously space and time-resolved plasma spectroscopy during transients, stable and turbulent regimes.
\end{abstract}

Keywords: X-ray Imaging; Plasma Diagnostics; Electron Cyclotron Resonance Ion Sources; High Dynamical Range Analysis; Single-Photon-Counted Images; X-ray Spatially-resolved Spectroscopy

\section{Introduction}

At the INFN-LNS and in collaboration with the ATOMKI Laboratories, efforts have been paid to the study, development and use of an innovative multi-diagnostic setup with advanced analytical techniques aiming at characterizing the thermodynamical properties of the ECR magnetized plasmas confined in compact traps for multidisciplinary studies. The developed multi-diagnostics setup [1,2] includes a microwave interfero-polarimeter, several RF multi-pins probes, a multi-X-ray detectors system for $X$-ray spectroscopy, a $X$-ray pin-hole camera for high-resolution 2D space resolved spectroscopy and different spectrometers for the plasma-emitted visible light characterization.

In the framework of the PANDORA_Gr3 (Plasmas for Astrophysics, Nuclear Decays Observation and Radiation for Archaeometry) project [3], the multi-diagnostic system will equip an innovative compact and flexible magnetic plasma trap for measuring nuclear 
$\beta$-decay rates of nuclear astrophysics interest, for the first time, in laboratory plasmas $[4,5]$. On the other hand, plasma diagnostics plays a crucial role for applications, i.e. for the development and the improvement of future ECR ion sources (ECRISs) able to produce beams of highly charged ions with high intensity and stability necessary for accelerators in applied and nuclear physics research.

Among the other techniques, the X-ray plasma diagnostics have been developed along the years allowing volumetric characterization of the ECR plasmas [6-10]. The volumeintegrated X-ray diagnostics in the soft-X domain (from $1 \mathrm{keV}$ to $30 \mathrm{keV}$ ) allow to probe the "warm" plasma population, which plays a crucial role in ionization and charge distribution build-up. Several experimental campaigns were carried out at INFN-LNS, at the GSIDarmstadt and at ATOMKI-Debrecen [11-14], measuring the warm electrons temperature and density by applying an emissivity model [15].

High precision X-ray measurements can be also performed in a spatially-resolved way by means of the powerful $X$-ray pin-hole camera tool which allows to perform high resolution spatially-resolved spectroscopy. Even if our teams have already been using the X-ray camera techniques combined with pinholes since more than 15 years [16-22], and this method has been routinely used in several other laboratories (for example at Z-machines [23-26]), recently both the tool [27] and the advanced analytical method have been drastically improved.

In this paper the on-purpose developed analytical method will be presented. A suite of MatLab codes has been developed from the ground, supporting the analysis of the $X$-ray pin-hole camera raw images. In particular, the algorithm is now able to analyse the Single Photon-Counting (SPC) images for energy-resolved investigation pixel-by-pixel, to investigate the local plasma emitted spectrum in a High-Dynamic-Range (HDR) mode, by distinguishing fluorescence lines of the materials of the plasma chamber ( $\mathrm{Ti}, \mathrm{Ta})$ from plasma (Ar) fluorescence lines; moreover, a very high energy and spatial resolution has been achieved (respectively of $300 \mathrm{eV}$ at $8.1 \mathrm{keV}$ and $500 \mu \mathrm{m}$ ) by means of a part of the developed code able to remove almost totally the readout noise, which typically affects measurements acquired with very low exposure times $t_{\exp }$ (several tens of milliseconds), as the ones taken in SPhC mode. As a consequence, this method allows a quantitative characterization of warm electrons population in the plasma (and its 2D distribution), to estimate local plasma density and spectral temperatures, to investigate plasma morphology and confinement dynamics in ECR plasma trap. The analytical method and tool can be useful also in other devices, i.e. for plasma fusion or applications.

\section{The Experimental Setup}

The X-ray pin-hole camera system is sketched in Figure 1a. The CCD X-ray camera (model Andor, iKon-M SO series) is made of $1024 x 1024$ pixels, with an optimal quantum efficiency in the range $0.5-20 \mathrm{keV}$. It was coupled to a $\mathrm{Pb}$ pin-hole (thickness $2 \mathrm{~mm}$, diameter $\Phi=400 \mu \mathrm{m}$ ) placed along the axis, facing the chamber from the injection flange. A Titanium window with $9.5 \mu \mathrm{m}$ of thickness was used to screen the CCD from the visible and UV light coming out from the plasma.

In order to work with the ECR-plasma heated at high power, the overall pin-hole system has been redesigned, including a multi-disks lead collimator for extra shielding of scattered radiation. The multi-disks collimator consists of two lead disks with same thickness $(1 \mathrm{~mm})$ and different diameter $\Phi(2 \mathrm{~mm}, 1 \mathrm{~mm})$. The lead pin-hole was placed in between the two lead disks, that were located at distances of $l_{1}=40 \mathrm{~mm}$ far from the pin-hole at the CCD side, and $l_{2}=6 \mathrm{~mm}$ far from the pin-hole at the plasma side. The optical magnification $M$ was optimized to be: $M=0.244$ (distance $_{\text {pinhole-CCD }}=232 \mathrm{~mm}$, distance $_{\text {pinhole-plasma centre }}=952 \mathrm{~mm}$ ). Other details about the setup are described in [27]. By this system the pin-hole camera was operated up to $200 \mathrm{~W}$ total incident power in the plasma, namely one order of magnitude higher compared to the previous measurements (where the pumping power was limited to $30 W[20,21]$ ). This way, both stable and 
turbulent plasma regimes (typically triggered above certain power thresholds [28,29]) have been investigated $[30,31]$, also studying new plasma heating methods $[32,33]$.

(a)

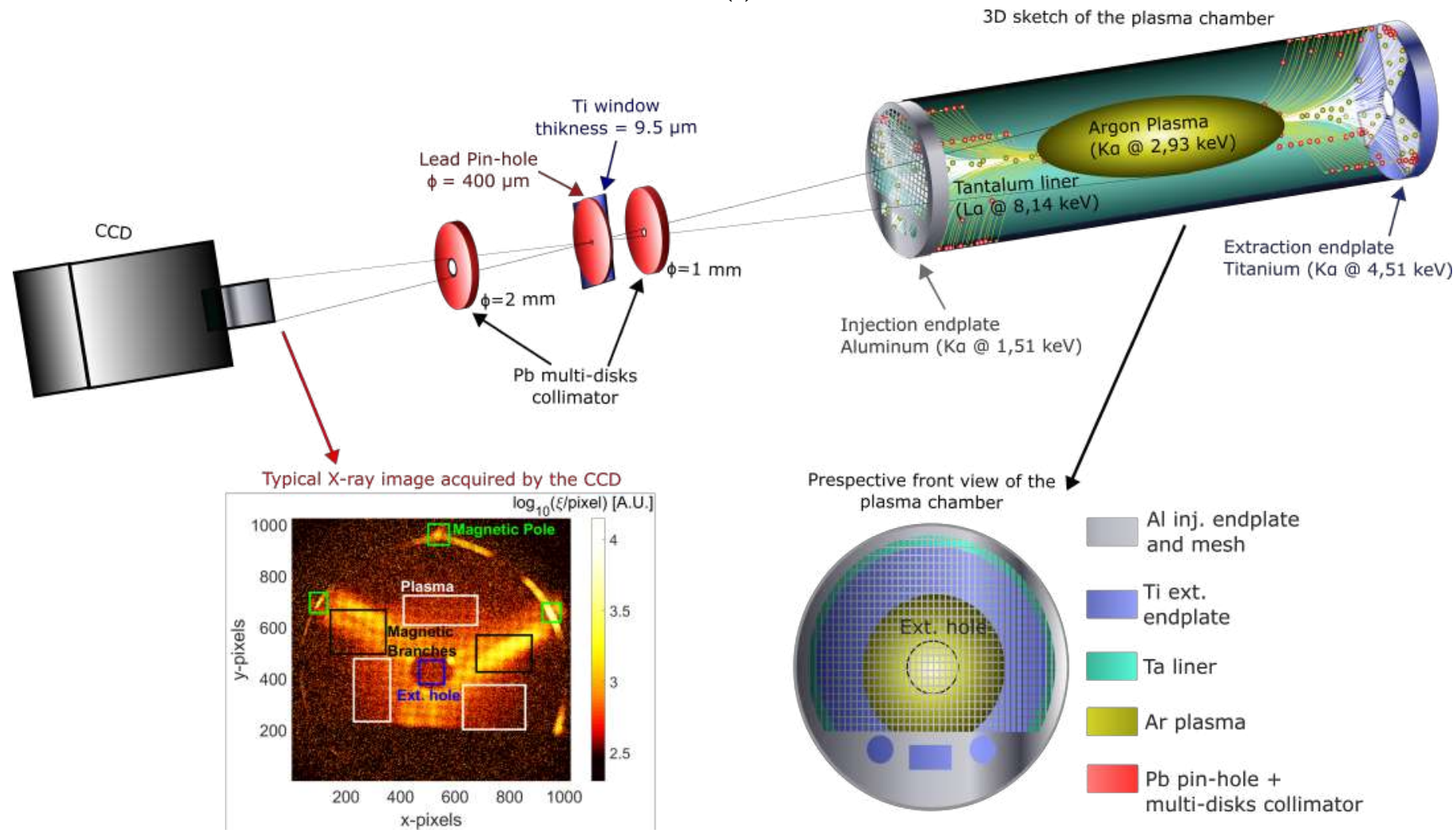

(b)

(c)

Figure 1. (a) Sketch of the X-ray pin-hole CCD camera system, including the multi-disks collimator. On the rigth a sketch of the ATOMKI plasma chamber highlighting the different materials and relative X-ray fluorescence peaks is shown: Al for the injection endplate mesh, Ti for the extraction electrode and Ta as a liner covering the lateral chamber walls. Ar was used to generate the plasma. The sketch also shows (in white) the magnetic field lines going from the plasmoid towards the chamber walls (both endplates and lateral): electrons are shown as small bullets with different colors, red for the ones impinging on the lateral walls while yellow for the ones flowing towards the endplates. (b) Pseudo-color elaboration of X-ray flux (in logarithmic scale), putting in evidence plasma branches (radiation coming from the extraction endplate), hot spots at the magnetic pole sites on the lateral chamber walls, the extraction hole and the plasma emission. (c) Perspective front view of the plasma chamber from the same side of the X-ray pin-hole camera.

In order to allow SPhC-based quantitative space resolved spectral analysis, the ATOMKI plasma chamber walls have been covered by thin layers of materials having different X-ray fluorescence energies, whilst the plasma was made by Ar, having a fluorescence emission around $2.96 \mathrm{keV}$. This way, even in energy integrated images, the X-rays coming from the plasma are well visible, while the materials of the extraction (Titanium) and injection (Aluminum) endplate and of the lateral walls (Tantalum) provide bright regions of X-ray emission due to bremsstrahlung and X-ray fluorescence as well.

The sketch of the ATOMKI plasma chamber is illustrated in the Figure 1a (on the right). This Figure reports in a pictorial way the shape of the plasma core, the so-called plasmoid, that is typically contained within the iso-magnetic surface fixed by the ECR condition $\omega_{R F}=q B / m$, where $\omega_{R F}$ is the RF pumping frequency, $B$ is the magnetic field, $q$ and $m$ the electron charge and mass, respectively. From this high density core, fluxes of deconfined electrons and ions escape according to the so called "loss cones". Those field lines lying 
inside the loss cones are represented in white. Small bullets of different colors represent the lost electrons, going towards the lateral chamber walls (red dots) or towards the endplate (yellow dots).

The perspective front-view of the plasma chamber (i.e. the same view of the pin-hole camera setup) is sketched in Figure 1c. It is clearly visible the Al mesh (having a wire diameter of $400 \mu \mathrm{m}$ ) that was placed on the injection endplate to keep the microwave resonator-like properties of the plasma chamber, but allowing direct inspection of the chamber interior at the same time, guaranteeing more than $60 \%$ of optical X-ray transmission.

\section{X-ray Spectrally-Resolved Imaging Algorithm}

Each photon impinging in the CCD camera releases its energy in the silicon generating a characteristic number of photo electrons that is proportional to its own energy. The Analogue Digital Unit (ADU), corresponding to the information read by the CCD in each pixel, is therefore proportional to the product of the incident photons by their energies. We adopted two operative modes to acquire the images: the Spectrally Integrated (SpI) mode and the SPhC mode.

Images obtained with long $t_{\exp }$ (tens of sec) will be called SpI images: in this case no energy discrimination is possible (but they are useful anyway, since the ADUs are related to the energy content of the plasma). These acquisitions are relatively fast and it is possible to monitor "on line" changes of the plasma structures or plasma losses on the chamber walls. A typical example of a SpI image is shown in Figure 1b. This image was collected with an exposure time of 50 seconds, at $1 \mathrm{MHz}$ of readout rate and using the full-frame (1024 $x 1024$ pixels) acquisition mode. ROIs (Regions of Interest) in the images were individuated according to the expected structure of the ECR plasma in $B-$ minimum traps, as sketched in Figure 1a. Figure $1 \mathrm{~b}$ shows several bright regions due to plasma and plasma chamber walls emission. The above mentioned ROIs, in the light of the detailed description of the Figure 1a, are here reported including:

- white rectangles, enclosing the regions where the X-rays come from the plasma region (i.e. there are no perspective interceptions with the chamber endplates, beyond of them);

- $\quad$ black squares, highlighting the so-called "magnetic branches", i.e. the bremsstrahlungproduced X-rays and X-ray fluorescence coming from extraction endplate, that are due to electrons escaping axially (in yellow in the pictorial view of Figure 1a);

- bright green squares, indicating the regions where the magnetic field lines intercept the lateral walls of the plasma chamber (the ones in red in Figure 1a).

This plasma image demonstrates the capability of this method to qualitatively separate $X$-ray radiation coming from the plasma from that one coming from the plasma chamber walls, but the more powerful investigations, able to perform local energy determination, is provided by $\mathrm{SPhC}$ mode.

To operate in SPhC mode the development of proper acquisition and post-processing procedures was required.

The SPhC mode is obtained by fixing a very short $t_{\text {exp }}$ for each of the acquired image-frame (several tens of milliseconds) minimizing the probability that two (or more) photons hit the same pixel or its nearest neighbours.

SPhC $t_{\exp }$ was empirically set-up, in such a way that only a few number of pixels were illuminated on the full CCD matrix during a single-frame acquisition; a sequential acquisition of thousands of SPhC frames then allows to reach the statistics necessary for obtaining high quality $\mathrm{X}$-ray fluorescence spectra and images.

The developed analytical post-processing method was developed in MatLab and it consists of five different steps:

(I) Grouping process (Gr-p);

(II) Energy Calibration and Event Counting Normalization (E-Cal and EC-Norm);

(III) Energy Filtering process (EF-p);

(IV) High Dynamical Range (HDR) imaging and spectroscopy (HDR-im and HDR-sp); 


\section{(V) ReadOut Noise (RON) removal (RON-r).}

The details of each step are described, respectively, in the next five subsections.

\subsection{The Grouping Process}

Even working in SPhC mode, some pixel-clusters are often in each of the image-frame acquired: they could be associated with a single photon that interacts with more than one pixel or with two (or more) photons hitting neighboring pixels. Grouping process (Gr-p) aims at finding real SPhC clusters, discarding spurious events.

On this purpose, we introduced the $S$ parameter representing the maximum cluster size (defined as the number of neighbours pixels in a cluster) that can be considered as due to a single-photon event. Clusters larger than $S$ are filtered out. Noise contribution is removed by setting a threshold $L$.

In more detail, the algorithm works in the following way:

STEP-1: considering a given image-frame $J$ : all pixels having ADU $<L$ are "turned off"; then, for a given $J$ frame, neighbouring pixels of an illuminated pixel at coordinates $(X, Y)_{J}$ are checked. In case of no-charges in the surrounding pixels, the event is considered purely single photon and it is associated with 3-coordinates $(X, Y, E)_{J}$, being $E$ the energy associated with the photon (determined as in section 3.2). See also Figure 2a;

STEP-2: when a group of active neighbouring pixels (a cluster) is identified, the algorithm tries to distinguish single from multiple-events:

(a) single photon-event clusters are typically characterized by a single, clear maximum: for them, in the STEP-3 described below we will see that the code will associate the integrated total charge to a single photon energy, whose $(X, Y)_{J}$ coordinate corresponds to the maximum;

(b) clusters associated with multiple-hit events (showing different relative maxima in a big-size cluster) are filtered out from SPhC images. However, the algorithm store the information anyway, labelling them as spurious events (in $\mathrm{SPhC}$ images their value is put to zero);

STEP-3: charges of all those pixels belonging to non-spurious clusters (see Figure $2 \mathrm{~b}$ ) are integrated in order to provide energy-position information of single-photon events (reported in Figure 2c).

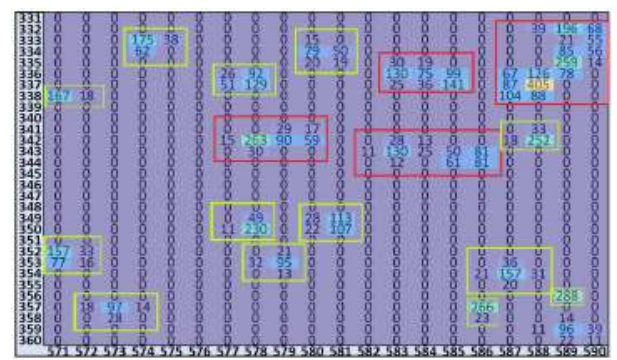

(a)

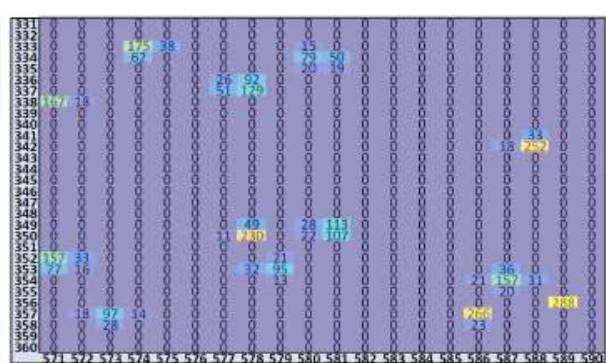

(b)

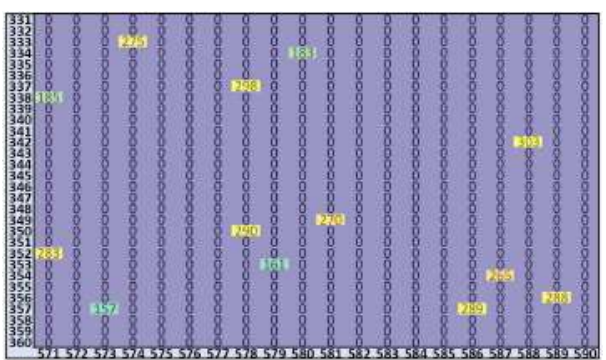

(c)

Figure 2. ROI of $20 X 30$ pixels analyzed implementing the three different steps (described in the text) of the Gr-p: a) after the STEP-1; b) after the STEP-2; c) after the STEP-3.

In Figure 2 a ROI of $20 \times 30$ pixels has been processed by Gr-p with $S=5$ and $L=10$ as input parameters. 


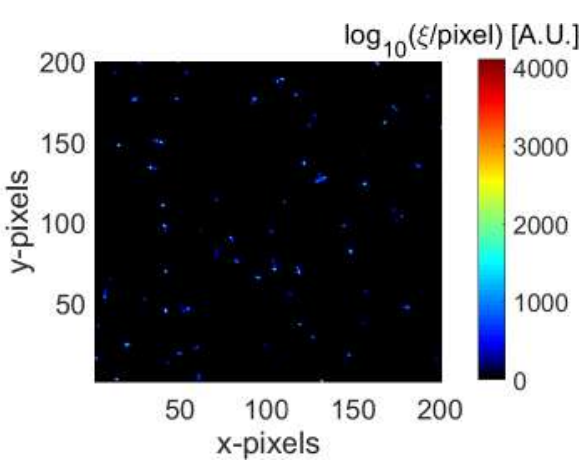

(a)

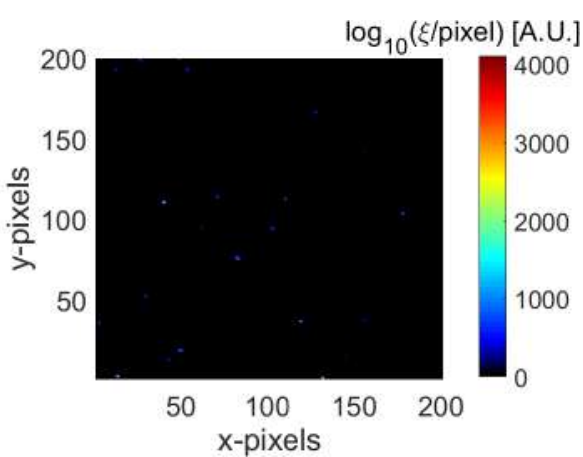

(b)

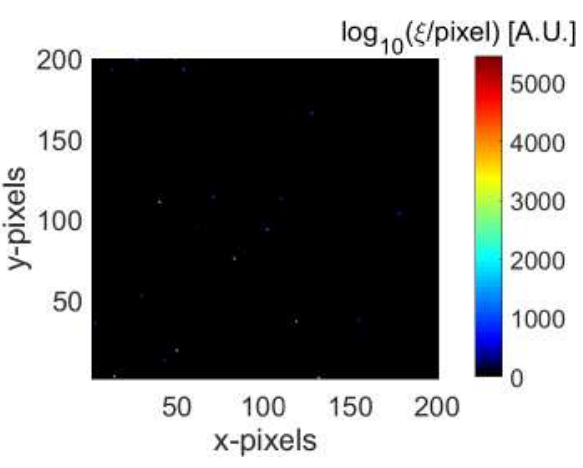

(c)

Figure 3. ROI of 200X200 pixels analyzed implementing the three different steps of the Gr-p: a) after the STEP-1; b) after the STEP-2; c) after the STEP-3.

Figure 3 reports the corresponding images (this time obtained considering a larger ROI of $200 \times 200$ pixels) after the application of the grouping algorithm.

After Gr-p process application to the whole data-set, $N$ becomes the number of photons with energy $E$ in the position $(X, Y)$. Therefore, we get the 4-dimensional array $(X, Y, E, N)$ is obtained. Integrating over the spatial coordinates $(X, Y)$ we get the $N_{\text {tot }}$ versus the energy E plot, i.e. the full X-ray spectrum. This latter is reported in Figure 4. The Figure shows the full field X-ray spectrum collected from a plasma obtained at $200 \mathrm{~W}$ of RF power and $13.90 \mathrm{GHz}$ of RF frequency.

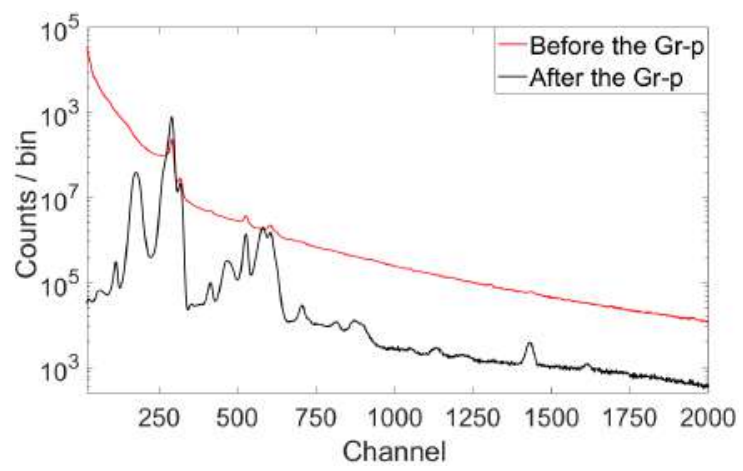

Figure 4. Full field X-ray spectrum (experimental configuration: RF power of $200 \mathrm{Watt}$, frequency $13.90 \mathrm{GHz}$ ). In red, the spectrum evaluated before implementing the Gr-p. In black, the spectrum evaluated after implement the Gr-p $(L=10$ and $S=5$ were used as processing parameters).

In this figure, the raw spectrum (red line) is compared with the Gr-p post-processed one (in black). Fluorescence lines of $\mathrm{Al}, \mathrm{Ti}$ and $\mathrm{Ta}$ (coming from plasma and plasma chamber walls) are visible only in the post-processed plot.

\subsection{Energy Calibration and Counting Normalization}

In order to perform the energy calibration, four characteristic peaks corresponding to the $T i_{K_{\alpha}}, T i_{K_{\beta}}, T a_{L_{\alpha}}$ and $T a_{L_{\gamma}}$ fluorescence peaks have been considered. The energy and channel values of each peak are summarized in the Table 1 and the linear correlation coefficient of the linear fit results $R=1$.

Table 1. Energy and channel values of the four characteristic fluorescence peaks chosen to perform the energy calibration.

\begin{tabular}{lllll} 
& $\mathbf{T i}_{\mathbf{K}_{\mathbf{a}}}$ & $\mathbf{T i}_{\mathbf{K}_{\mathbf{b}}}$ & $\mathbf{T a}_{\mathbf{L}_{\mathbf{a}}}$ & $\mathbf{T a}_{\mathbf{L}_{\mathbf{g}}}$ \\
\hline Channel & 290 & 318 & 527 & 705 \\
\hline Energy & 4.51 & 4.93 & 8.14 & 10.96 \\
\hline
\end{tabular}


The calibrated spectrum, expressed in logarithmic scale, is shown in Figure 5a, whilst a zoom in the energy range $1-15 \mathrm{keV}$, where all characteristics peaks are highlighted by labels, is shown in Figure 5b. Fluorescence peaks (the $K_{\alpha}$ and $K_{\beta}$ of Titanium, the $L_{\alpha}, L_{\beta}$, $L_{\gamma}$ and $M_{\alpha}$ of Tantalum) and of the plasma ( $K_{\alpha}$ and $K_{\beta}$ of Argon) have been highlighted by appropriate labels.

The estimated energy resolution is $0.326 \mathrm{keV}$ at $8.1 \mathrm{keV}$ (corresponding to the $T a_{L_{\alpha}}$ line).

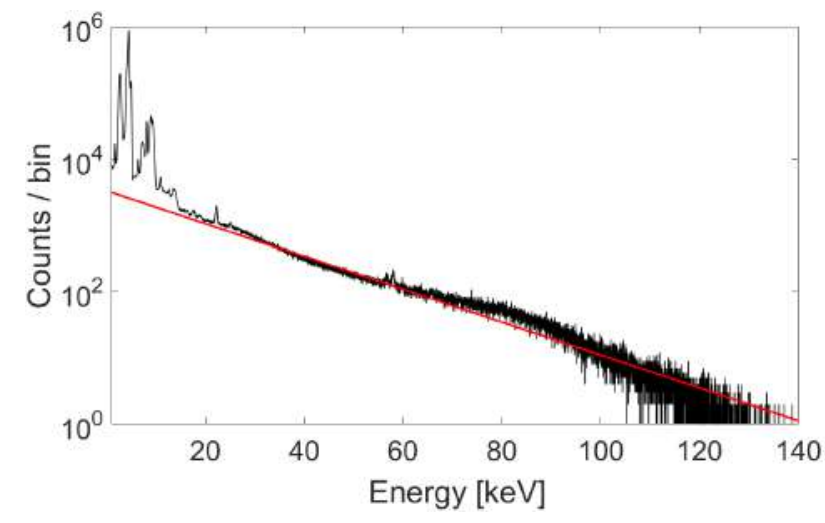

(a)

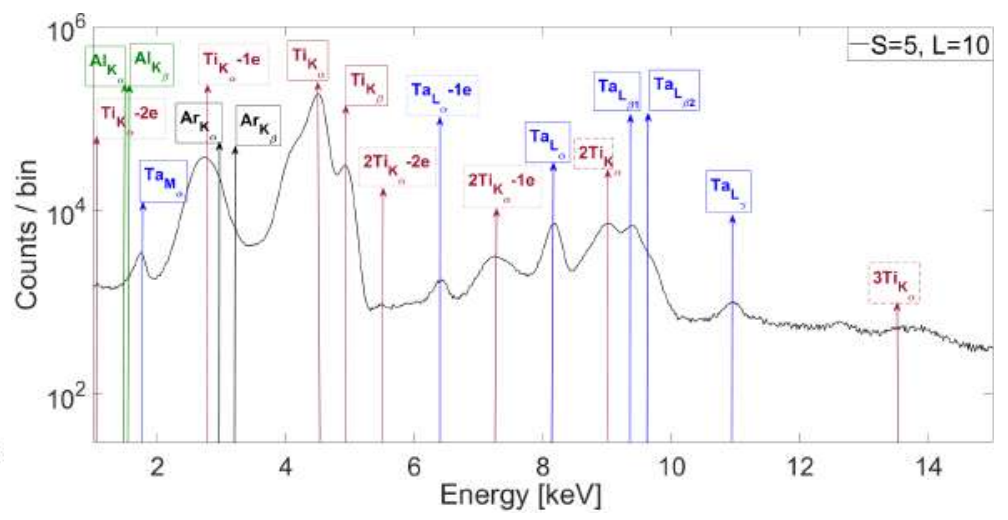

(b)

Figure 5. (a) Typical X-ray spectrum expressed in logarithmic scale after energy calibration, the red line is the exponential fit performed in the $30-100 \mathrm{keV}$ range. (b) Typical calibrated X-ray spectrum with the labels of each characteristic peak.

Dimer and escape peaks of the silicon-made CCD are also visible. This latter peak is an artifact, due to the probable $S i_{K \alpha}$ X-ray photons generation inside the detector: these photons can escape from the detector volume, thus reducing the effective detected energy of some incident rays by $\sim 1.74 \mathrm{keV}$ ( $S i_{K \alpha}$ X-ray energy). The escape peak coming from Ti fluorescence overlaps with the $A r_{K \alpha}$ fluorescence. Consequently, methods to minimize these contributes have been also implemented. Several papers [34-36] demonstrated that the escape peak generation efficiency is typically of the order of $3 \%$ of the main $K \alpha$ peak. So, the algorithm considers an escape peak efficiency $Z$ of the $3 \%$ of the main $T i_{K \alpha}$ peak, and removes this contribution pixel-by-pixel both in the whole matrix and in the spectrum. Moreover, in order to remove the Bremsstrahlung continuum radiation, generated by the deceleration of the energetic electrons colliding with heavy ions in the plasma and with the atoms of the plasma chamber walls, an exponential fit has been performed, in the range 30 - $100 \mathrm{keV}$ (it corresponds to the red line in Figure 5a).

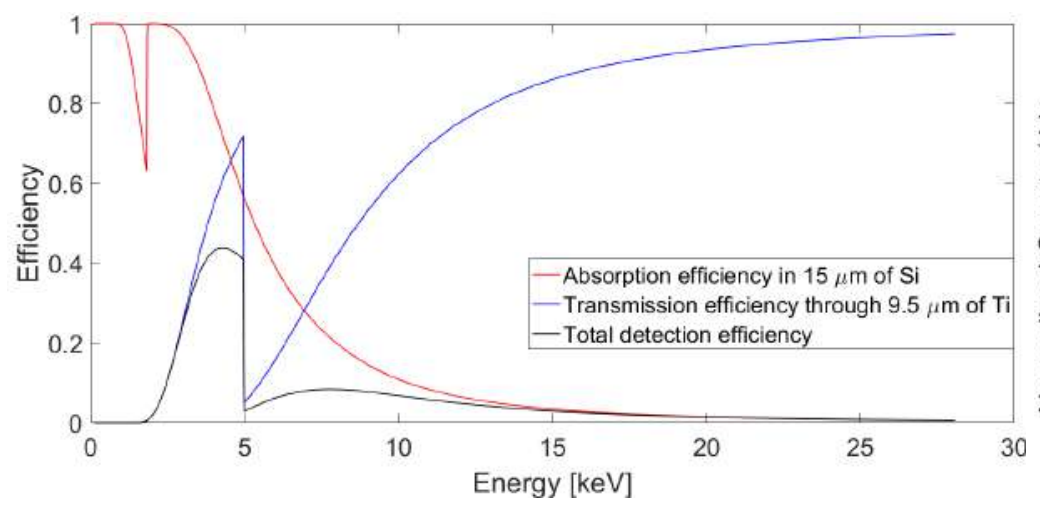

(a)

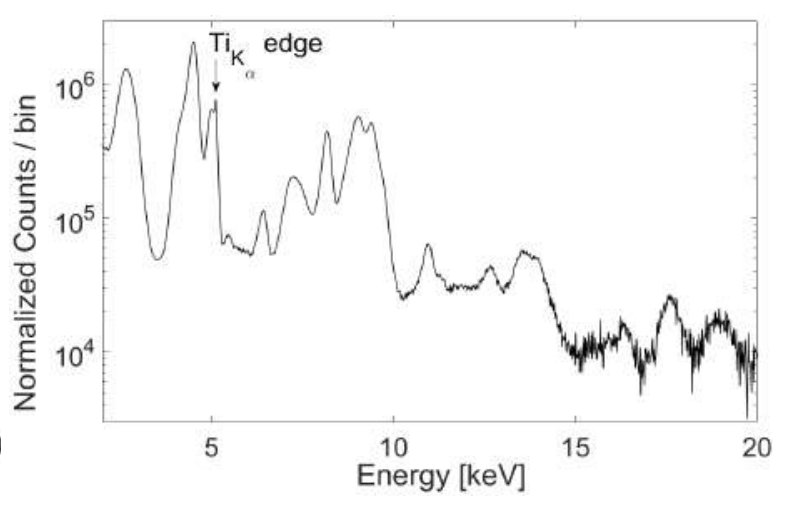

(b)

Figure 6. (a) Q.E. of the CCD camera (red), transmission efficiency through the Titanium windows (blue), total detection efficiency (black), curves. (b) Typical event count normalized and energy calibrated spectrum.

Finally, spectra need to be renormalized including both the Quantum Efficiency (Q.E.) of the CCD camera (absorption efficiency in $15 \mu \mathrm{m}$ of $\mathrm{Si}$ ) and the transmission efficiency 
through the Titanium windows (9.5 $\mu \mathrm{m}$ of $\mathrm{Ti}$ thickness): this has been done by means the NIST database [37]. The total detection efficiency, called $F(E)$, has been obtained multiplying the Quantum and the Transmission efficiencies. The three curves are shown in Figure 6a. All event counts have been normalized, dividing them for $F(E)$. An example of event count normalized spectrum is shown in Figure 6b.Effect of the renormalized eventcounting to the image matrix can seen in Figure 9.

\subsubsection{Optimization of the Algorithm}

Spectrum shape and intensity change as a function of $L$ and $S$ values have been optimized trying to reach a trade-off between spectral resolution and high enough statistics. In the Figure 7 a five different spectra evaluated by setting different $S$ values (from 1 up to 5 ), fixing $L=10$, are shown (in the energy range $1-15 \mathrm{keV}$ ). The spectra in the whole energy range are instead shown in Figure $7 \mathrm{~b}$.

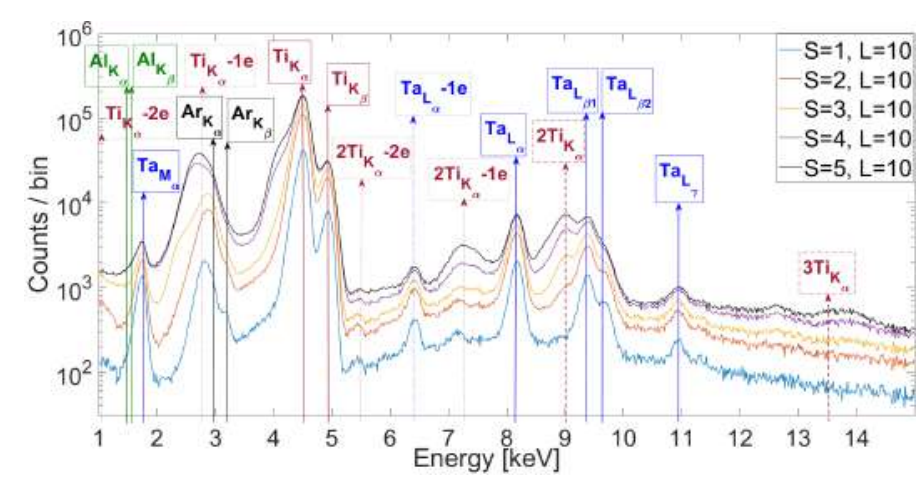

(a)

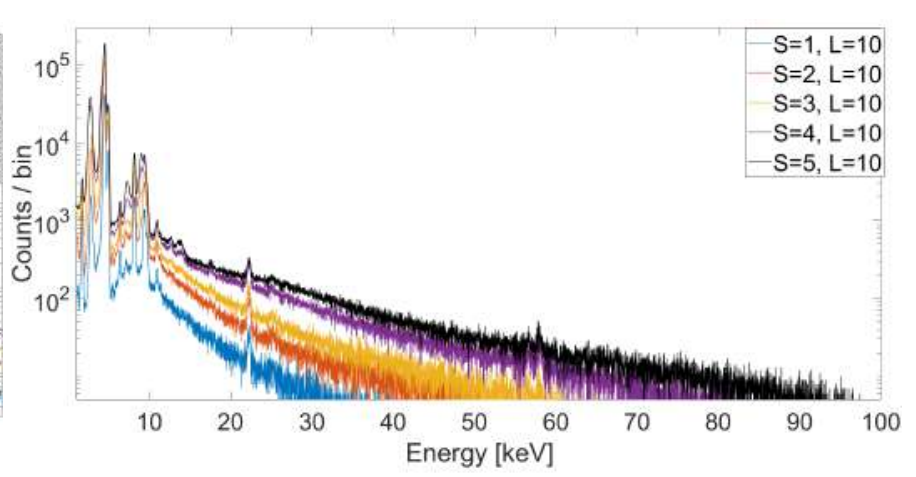

(b)

Figure 7. X-ray spectra evaluated by setting five different $S$ values (from 1 up to 5 ) and fixing $L=10$ : (a) in the energy range $1-15 \mathrm{keV}$ and (b) in the whole energy range.

It comes out that at the lowest $S$ the spectrum shows the higher resolutions but the lowest intensities. In addition, since at the lowest $S$ we have an overfiltering of larger clusters, the maximum energy (consequently, the electron temperature that is inversely proportional to the spectrum slope [15]) decreases as a function of $S$, as shown in the Figure $7 \mathrm{~b}$.

To account for these effects, and also to reduce the impact of dimer and escape peaks, the implemented algorithm optimizes the input $S$ and $L$ parameters (we set three $L=5,10,15$ values: for $L=5$ and $L=15$ the code run for five values of $S=1-5$, for the case $L=10$ we have $S$ from 1 to 10 ) for improving as much as possible the $\frac{I_{M P}}{I_{E P}}$ and $\frac{I_{M P}}{I_{D P}}$ ratios (where $I_{M P}, I_{E P}$ and $I_{D P}$ are, respectively, the main peak intensity, the escape peak intensity and the dimer peak intensity).

Optimization was searched by a trade-off between overall statistics and minimization of spurious peaks (escape, dimer, triplet, etc.).

For each configuration the following three values have been estimated:

a) the $\frac{I_{M P}}{I_{E P}}$ ratio for $T a_{L_{\alpha}}$;

b) the $\frac{I_{M P}}{I_{D P}}$ ratio for $T i_{K_{\alpha}}$;

c) the total $I_{M P}$ for $T i_{K_{\alpha}}$;

From the analysis results that:

a) the escape peak intensity vs. the main peak one is almost independent of $L$ and $S$;

b) the dimer peak intensity dramatically increases with respect to the main peak when increasing the clusters size;

c) the intensity of the spectral components (e.g. $I_{T i_{K \alpha}}$ ) increases with $S$. 
By adding the same relative weight of the three optimization procedures (low escape, low dimer, high intensity), the optimal values were: $L=10$ and $S=2$. In case of low statistic, $L=10$ and $S=5$ resulted to be the best compromise.

In summary, the whole process described in the previous sections (i.e. from raw data to calibrated and optimized spectra) is summarized in the sketch shown in Figure 8.
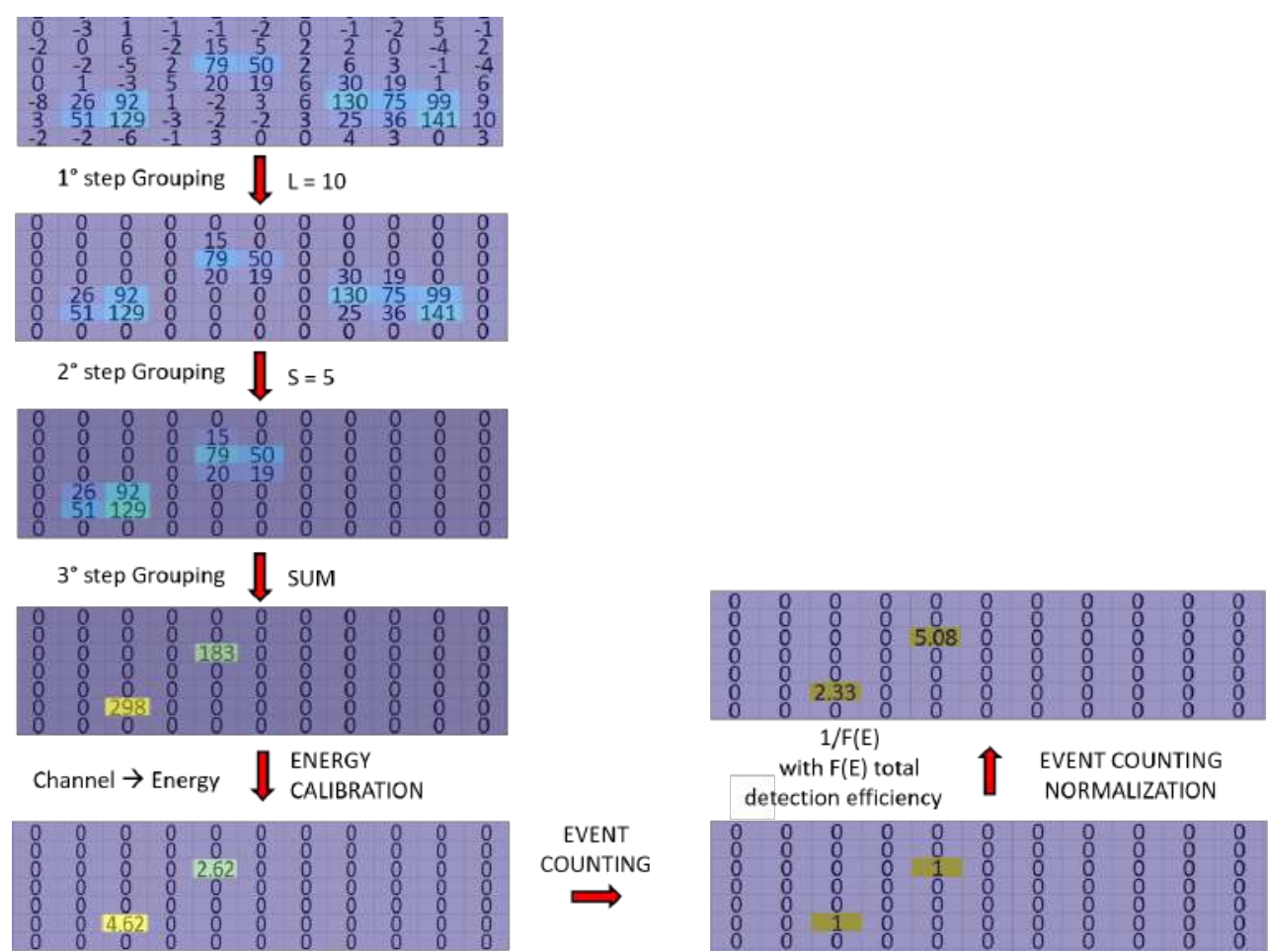

Figure 8. Summary of the implemented procedure of post-process image analysis, considering a sub-matrix of [7x11] pixels. Left, from top to bottom - Raw image; post-processed image after the 1st Gr-p step; post-processed image after the 2nd Gr-p step; post-processed image after the 3rd Gr-p step; energy calibrated image. Right, from bottom to top - Event counted image; event counted and normalized image.

\subsection{Energy Filtered Images}

Let us now consider, for sake of example, the image shown in Figure 9a. The image is the result of the analysis of a number of image-frames $N_{f r}=4200$ acquired with $t_{\text {exp }}=500 \mathrm{~ms}$, at $1 \mathrm{MHz}$ of readout rate and full-frame (1024x1024 pixels) acquisition mode. The spectrum of the full size image is the one shown in the Figure 7a (for $S=5$ and $L=10$ ). The Energy-filtered Imaging consists in selecting only those pixels which were loaded by only those photons having an energy in a given energy interval $\Delta E$.

We plotted images using $\Delta E$ corresponding to the fluorescence peaks of $\mathrm{Ar}$, $\mathrm{Ti}$ and $\mathrm{Ta}$, respectively, in Figure 9b, 9c and 9d.

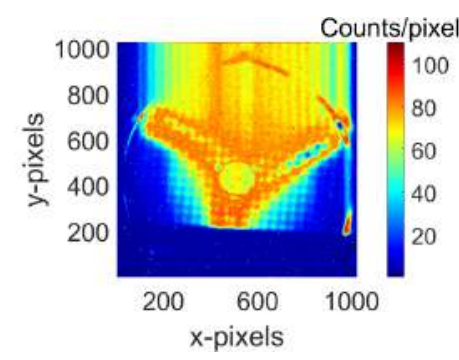

(a) Energy unfiltered image

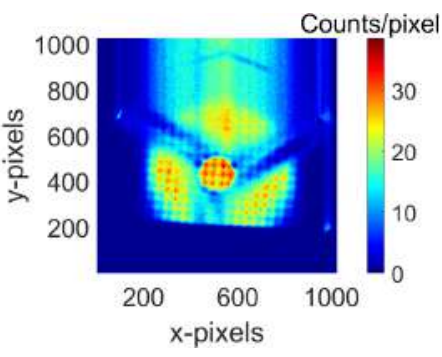

(b) Ar-energy filtered image

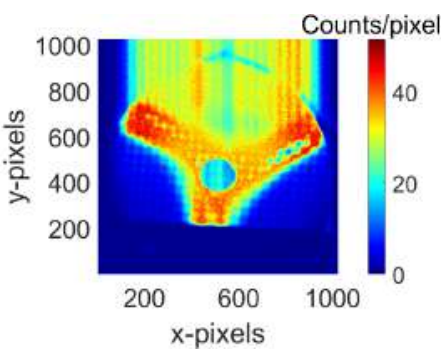

(c) Ti-energy filtered image

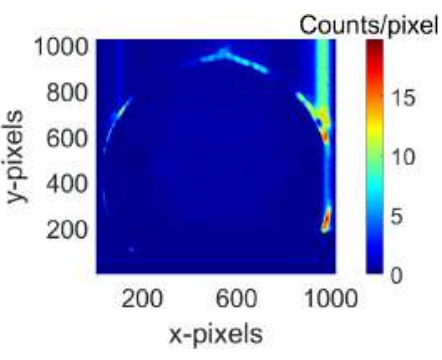

(d) Ta-energy filtered image

Figure 9. 2D SPhC images showing fluorescence photons coming from the Argon plasma and signals coming from the metallic layers covering plasma chamber walls. SPhC images were collected by the analysis of 4200 image-frames acquired with $t_{\text {exp }}=500 \mathrm{~ms}$, each using the full-frame (1024x1024 pixels) acquisition mode. 
In other words, and looking to the structure of the plasma and chamber depicted in Figure 1a, the images in Figure 9 display the emission coming from Ar plasma only, the effect of electrons lost by the magnetic confinement on the radial walls is visible through the fluorescence of Ta, while the axial losses are visible through the fluorescence of Ti.

\subsection{High Dynamical Range (HDR) Imaging and Spectroscopy}

When local emissivities of an image vary even of orders of magnitude, it is very challenging to determine the correct $t_{\text {exp }}$ in order to avoid extra or under-exposure. The solution comes from the HDR analysis - commonly used in visible light imaging - which consists in acquiring the same image setting two or more different $t_{\text {exp }}$ and then producing a weighted convoluted image.

In our case, SpI images at $t_{\text {exp }}=50$ s (see Figure 1b) display Ar intensities orders of magnitude lower than magnetic branches or poles. The same happens in SPhC mode.

In this latter case, we optimized $t_{\exp }$ and $N_{f r}$ acquiring different datasets, taking into account the hugely different local emissivities. In particular (see Table 2 ), we used $t_{\exp }=$ $0,5 s$ that was suitable to have good photon counting changes in the low intensity ROIs, acquiring a total of 4200 frames. These images were called I Plasma.

$t_{\text {exp }}=0,05 \mathrm{~s}$ and 1000 frames were instead set to have optimal SPhC in ROIs showing higher emissitivities: these ones were called $I_{\text {Pole }}$.

Table 2. Two acquisition modes, $I_{\text {Plasma }}$ and $I_{\text {Pole }}$, with different exposure times and number of frames.

\begin{tabular}{lll}
\hline Acquisition & $t_{\exp }[\mathbf{s}]$ & $\mathbf{N}$ frames \\
\hline$I_{\text {Plasma }}$ & 0,5 & 4200 \\
\hline$I_{\text {Pole }}$ & 0,05 & 1000 \\
\hline
\end{tabular}

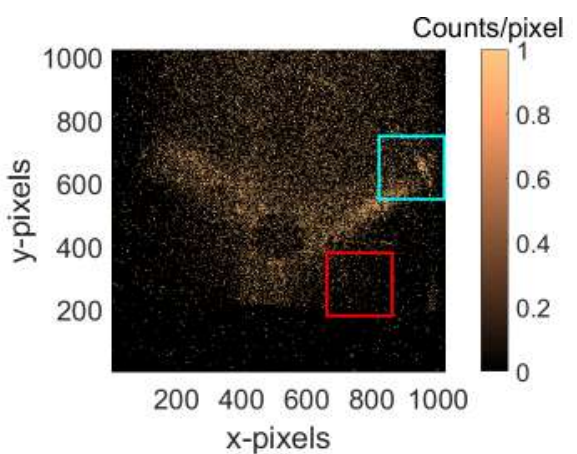

(a) I Ilasma : full-frame

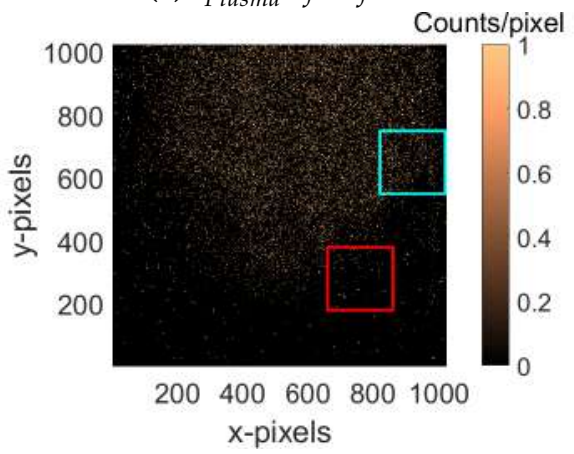

(d) $I_{\text {Pole }}$ : full-frame

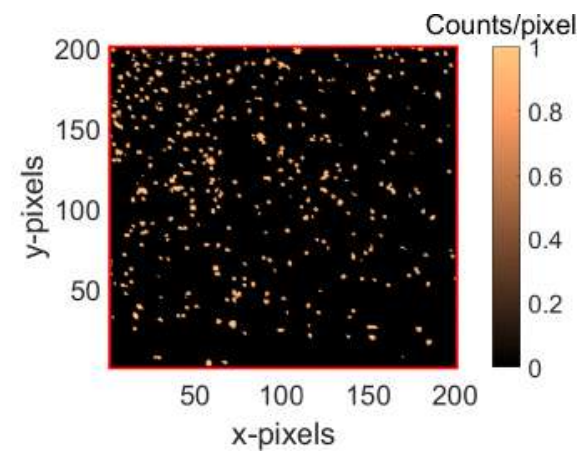

(b) $I_{\text {Plasma }}: R O I_{\text {plasma }}$

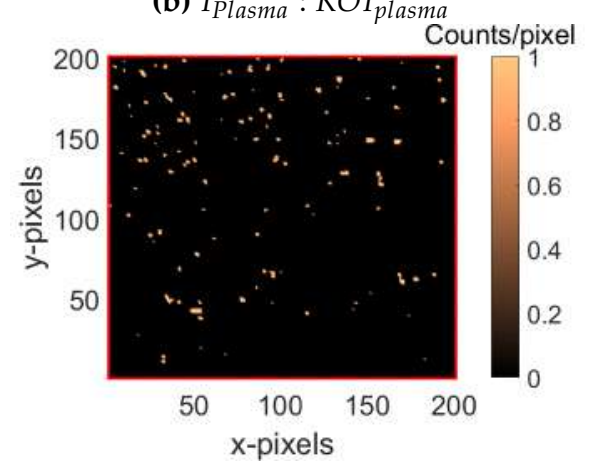

(e) $I_{\text {Pole }}: R O I_{\text {plasma }}$

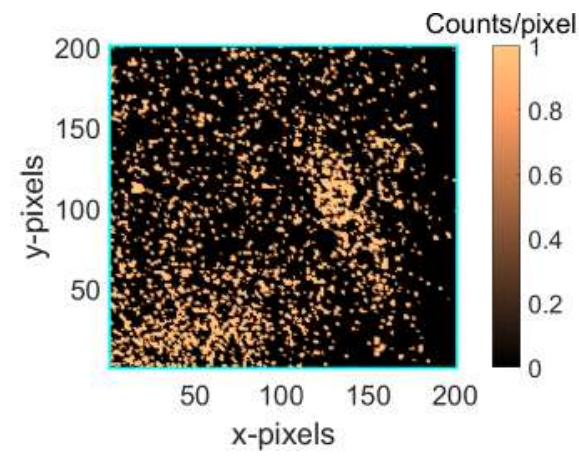

(c) $I_{\text {Plasma }}:$ ROI $I_{\text {pole }}$

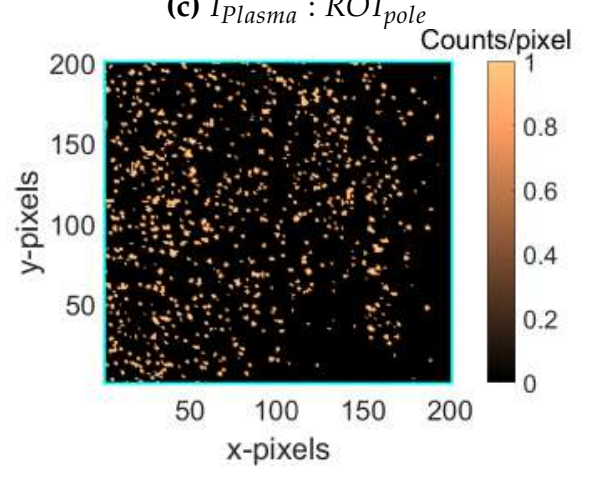

(f) $I_{\text {Pole }}: R O I_{\text {pole }}$

Figure 10. On the top, the $I_{\text {Plasma }}$ frame. On the bottom, the $I_{\text {Pole }}$ frame. a), d) - Event-counted single full-frame before applying the Gr-p. b), e) - image in the plasma ROI. c), f) - image in the magnetic pole ROI. 
As an example, two event-counted single full-frames (before applying the Gr-p) are shown in Figure $10 \mathrm{a}$ and $10 \mathrm{~d}$ for $I_{\text {Plasma }}$ and $I_{\text {Pole }}$, respectively.

It is possible to notice the difference from $I_{\text {Plasma }}$ and $I_{\text {Pole }}$ considering two ROIs:

- the plasma ROI: red squared in Figure 10a, 10d, where the zoom-in is shown in the images $10 \mathrm{~b}$ and $10 \mathrm{e}$ for $I_{\text {Plasma }}$ and $I_{\text {Pole }}$, respectively. In this ROI both the $I_{\text {Plasma }}$ and the $I_{\text {Pole }}$ frame are photo-counted, but only $I_{\text {Plasma }}$ frame shows high enough statistic, whilst in the $I_{\text {Pole }}$ frame it is too low.

- $\quad$ the magnetic pole ROI: cyan square in Figure 10a, 10d, where the zoom-in is shown in the images $10 \mathrm{c}$ and $10 \mathrm{f}$ for $I_{\text {Plasma }}$ and $I_{\text {Pole }}$, respectively. In this ROI the $I_{\text {Plasma }}$ frame is not photon-counted, whilst the $I_{\text {Pole }}$ frame is photon-counted and the loaded clusters are well isolated.

After applying the Gr-p and summing over the whole event-counted frames, the images shown in Figures $11 \mathrm{a}$ and $11 \mathrm{~b}$, for $I_{\text {Plasma }}$ and $I_{\text {Pole }}$ acquisition mode respectively, have been obtained (the frames have been also Ar-fluorescence energy-filtered). As expected, the $I_{\text {Plasma }}$ image shows a lack of information in the magnetic branches and poles ROIs. The correspondent spectra (called basic spectra (BS)) normalized by $t_{\text {exp }}$ and $N_{f r}$ are shown in Figure 11c. The $I_{\text {Plasma }}$ spectrum shows some missing (and under-represented) information too. Here the total rate (CPS, counts per second) is about one order of magnitude lower than the total rate (CPS) of $I_{\text {Pole }}$. In this condition one necessitates to combine two images and spectra into a single image and spectrum, respectively, by means of the High Dynamical Range procedure.

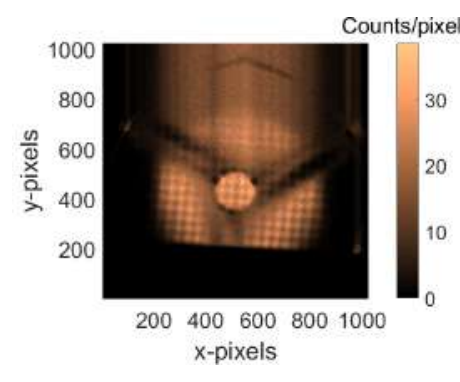

(a)

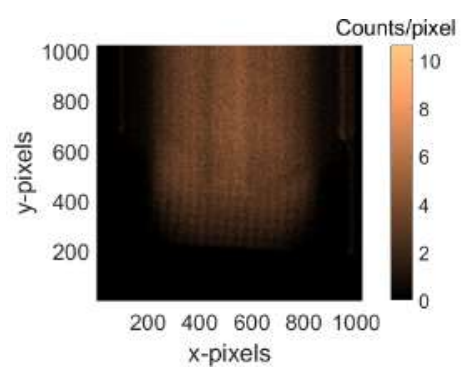

(b)

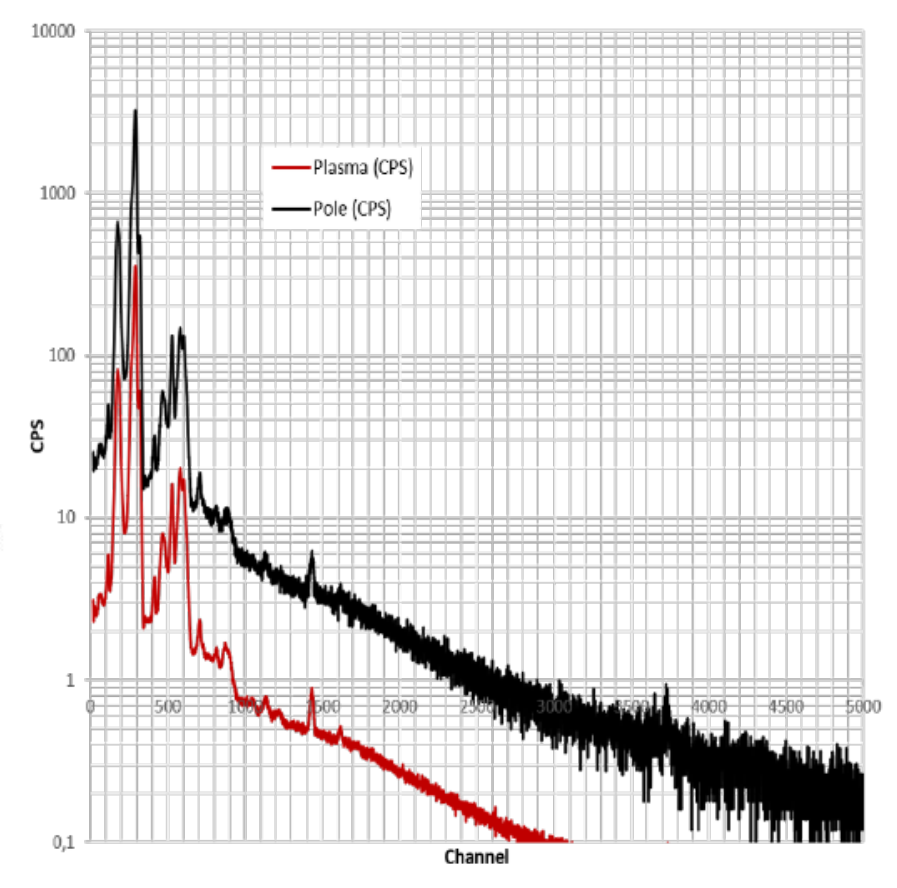

(c)

Figure 11. a) - Image acquired setting $t_{\text {exp }}=0,5 s$ and analyzing 4200 Ar-energy filtered image-frames. b) - Image acquired setting $t_{\text {exp }}=0,05 s$ and analyzing 1000 Ar-energy filtered image-frames. c) - Correspondent spectra to image a) and b) showing the total counts, expressed in counts per second (CPS).

Since the degree of the missing information is spatial-dependent, the implemented algorithm operates by exploring any pixel in each image-frame, as done for the Gr-p, but in this case the goal is to define two "masks" that are necessary to obtain the HDR image:

- HDR Mask: this mask shows those pixels which were not photon-counted on $I_{\text {Plasma }}$ images. It consists in a digital (0-1) array where position evaluated as 1 correspond to those pixels being part of a cluster larger than the $S$ parameter, for each image-frame. 
The sum of those images, divided by $N_{f r}$, gives the desired HDR Mask, where each number corresponds to the probability $\rho_{p l}^{n-p h c}$ normalized to 1 of a given pixel to be not photon-counted, as shown in the Figure 12a.

- Pole normalization Mask generation from $I_{\text {Pole }}$ frames: even the $I_{\text {Pole }}$ frames are not always photon-counted, therefore the counts are under-represented and the missing information is spatial dependent. Using the not-photon-counted matrix (NPhC) obtained from the $I_{\text {Pole }}$ images $\left(\rho_{\text {pol }}^{n-p h c}\right)$, a normalization matrix has been obtained (shown in the Figure 12b): Normalization matrix=1/(1-NPhC/1000).

- $\quad H D R$ image $=I_{\text {Plasma }}+I_{\text {Pole }} \cdot \mathrm{HDR}$ mask $\cdot$ Pole normalization mask. Of course, $I_{\text {Pole }}$ has been normalized for $t_{\exp }$ and $N_{f r}$. The HDR image is shown in the Figure 13.

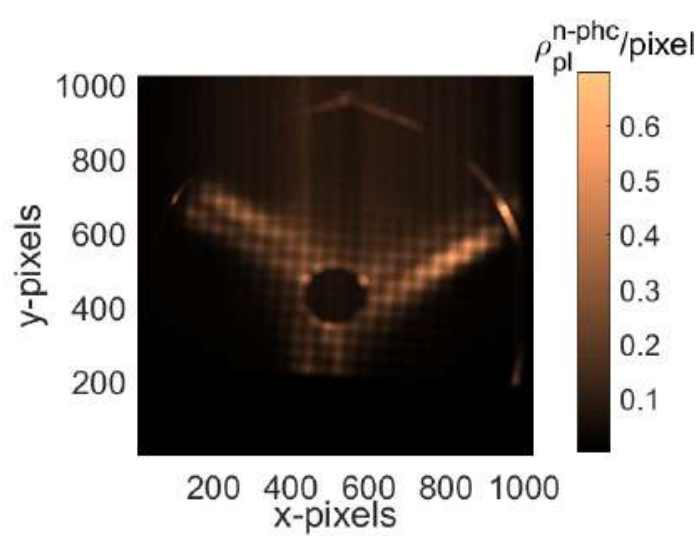

(a) HDR Mask

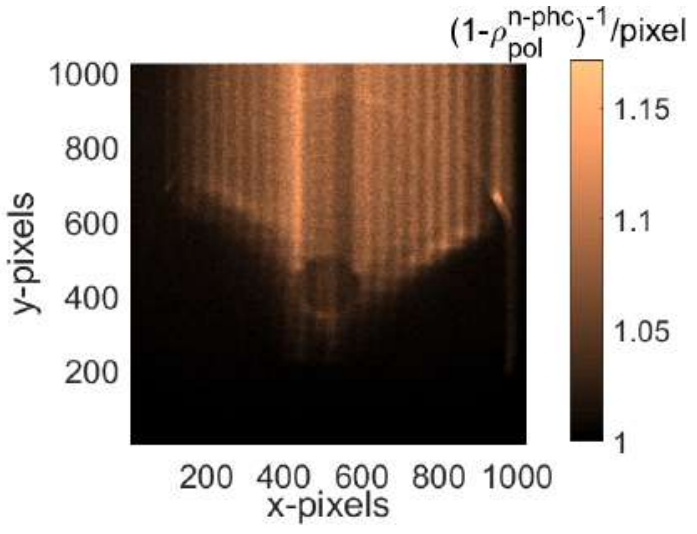

(b) Pole normalization Mask

Figure 12. a) - HDR Mask, showing space-resolved probability to find pixels which were not photon-counted on $I_{\text {Plasma }}$. b) Pole normalization Mask, $1 /(1-\mathrm{NPhC} / 1000)$, where NPhC is the not-photon-counted matrix obtained from the $I_{\text {Pole }}$.
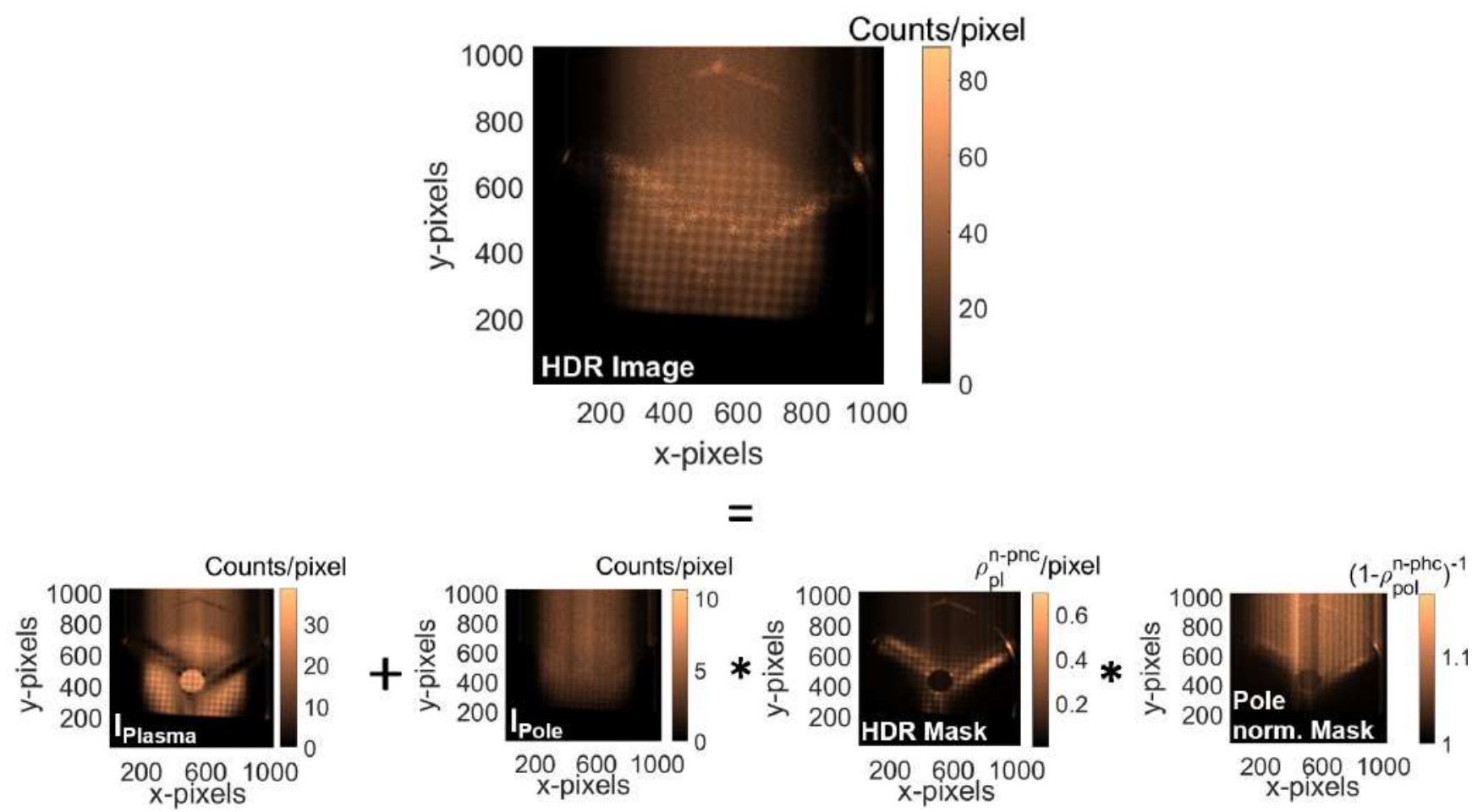

Figure 13. Top - HDR Image. Bottom - Procedure of multiplications and sums of matrices $\left(I_{\text {Plasma }}+I_{\text {Pole }}{ }^{*}\right.$ HDR mask ${ }^{*}$ Pole normalization mask) to obtain the HDR Image. 
Observing the Figure 13, it is clear that the HDR image - compared to the $I_{\text {Plasma }}$ and $I_{\text {Pole }}$ - contains all the information, with a good statistic both in the plasma, magnetic branches and poles ROIs. Pixels clusters that in $I_{\text {Plasma }}$ were filter out by the Gr-p, have been in fact replaced by the ones taken from the $I_{\text {Pole }}$, once weighted by the HDR and Pole normalization masks. Of course, the same analysis can be performed also in the energyunfiltered image and in the other energy-filtered images (for Titanium and Tantalum), as shown in Figure 14.

Finally, a similar HDR procedure has been applied to the basic spectra too.

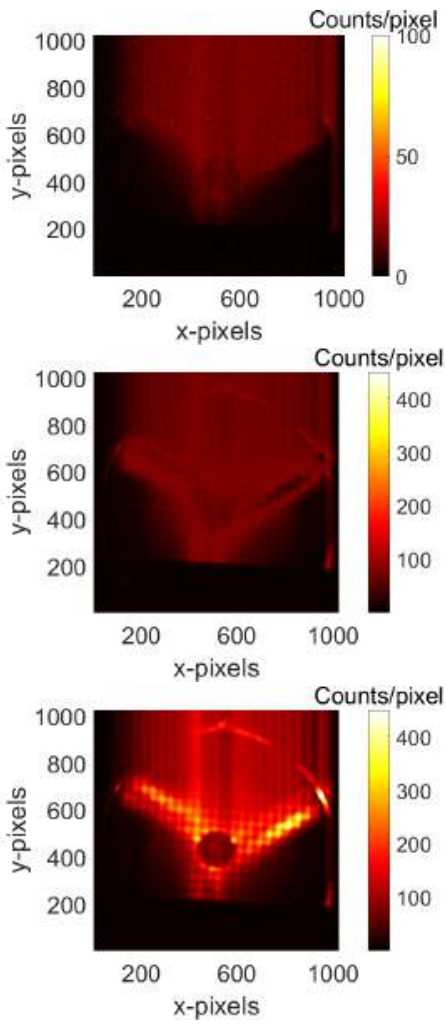

(a) Energy-unfiltered image

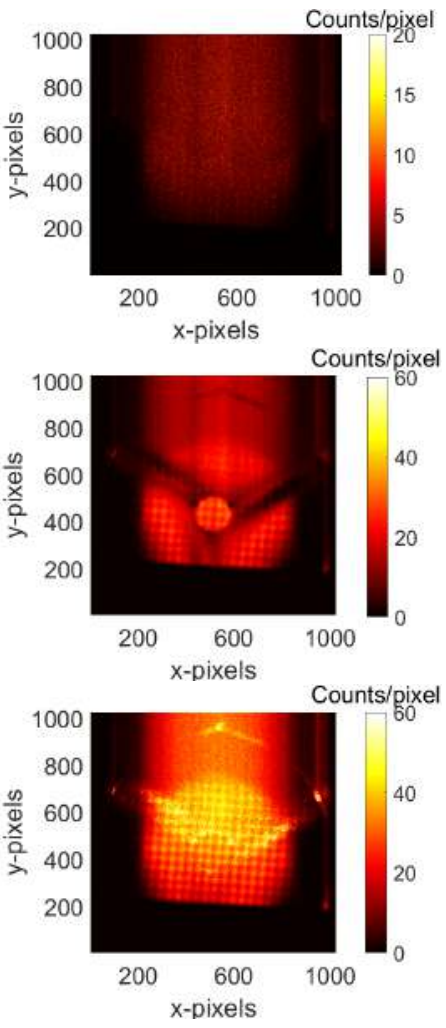

(b) Ar-energy filtered image

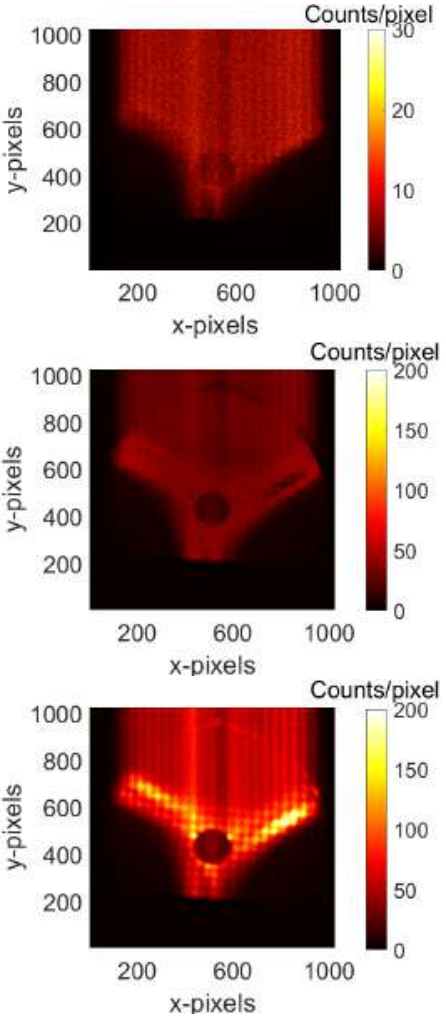

(c) Ti-energy filtered image

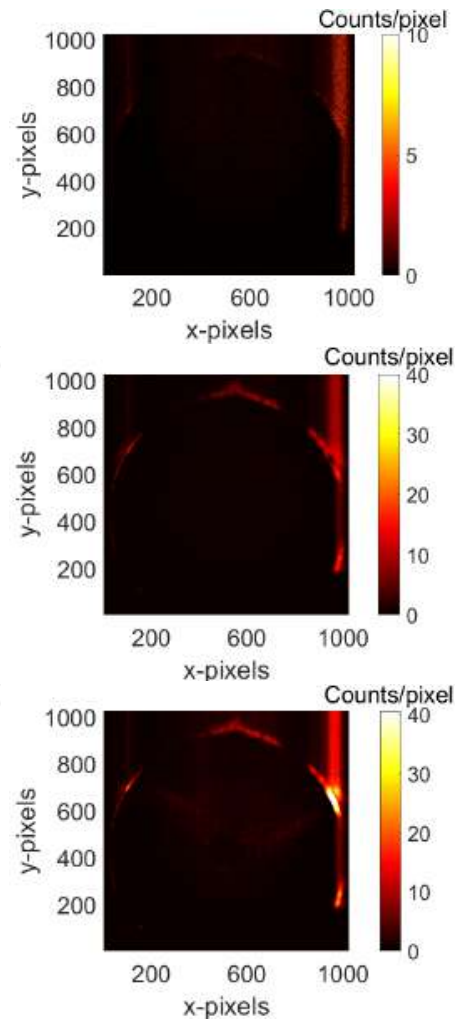

(d) Ta-energy filtered image

Figure 14. Top: the $I_{\text {Pole }}$ acquired images, for the energy ulfiltered case (a) and for each of the fluorescence contributions from $\mathrm{Ar}(\mathrm{b}), \mathrm{Ti}$ (c) and Ta (d). Middle: the $I_{\text {Plasma }}$ acquired images for the same cases of the row above. Bottom: the same of the rows above, but after HDR post-processing.

\subsection{The Readout Noise (RON) Removal Algorithm}

During the readout time $t_{R O}$ of the CCD camera, the accumulated charges are transferred through the rows of the CCD from the top to the bottom, where the reading buffer is placed. During this transition, the CCD is still active, so it is sensitive to the impinging photons. This implies that during the readout some photons are still detected, but in a "wrong place"; this effect causes the vertical "strips" visible in the images, as it is possible to observe in the Figures 14. If the $t_{R O} \sim t_{\text {exp }}$, the readout noise $(\mathrm{RON})$ is comparable to the real signal. Thus, its contribution has to be managed: removed or - if possible - recovered, since the acquisition even during the readout process is genuine despite being mismatched in position. A proper algorithm to handle this effect was written and applied. 

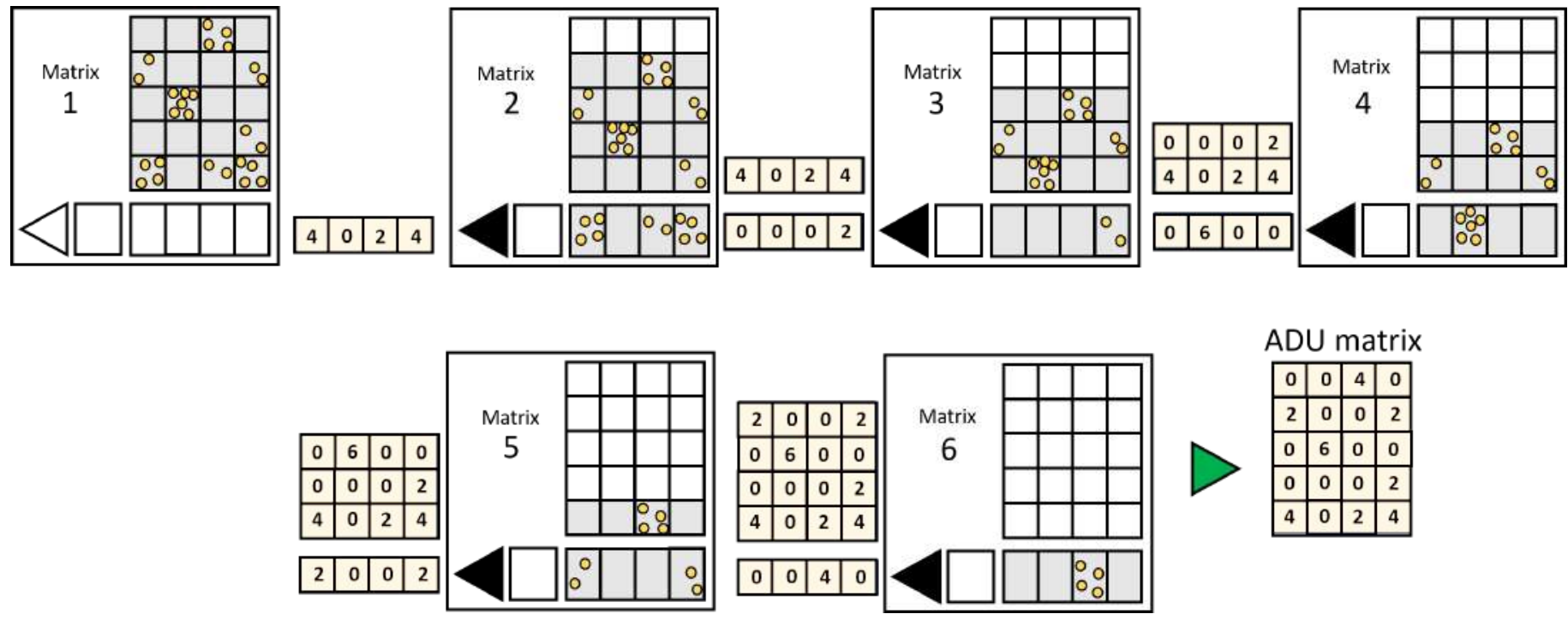

ADU matrix

Figure 15. Sketch of the readout procedure, neglecting the RON contribution. The numbers in the matrix show the total loaded charge.
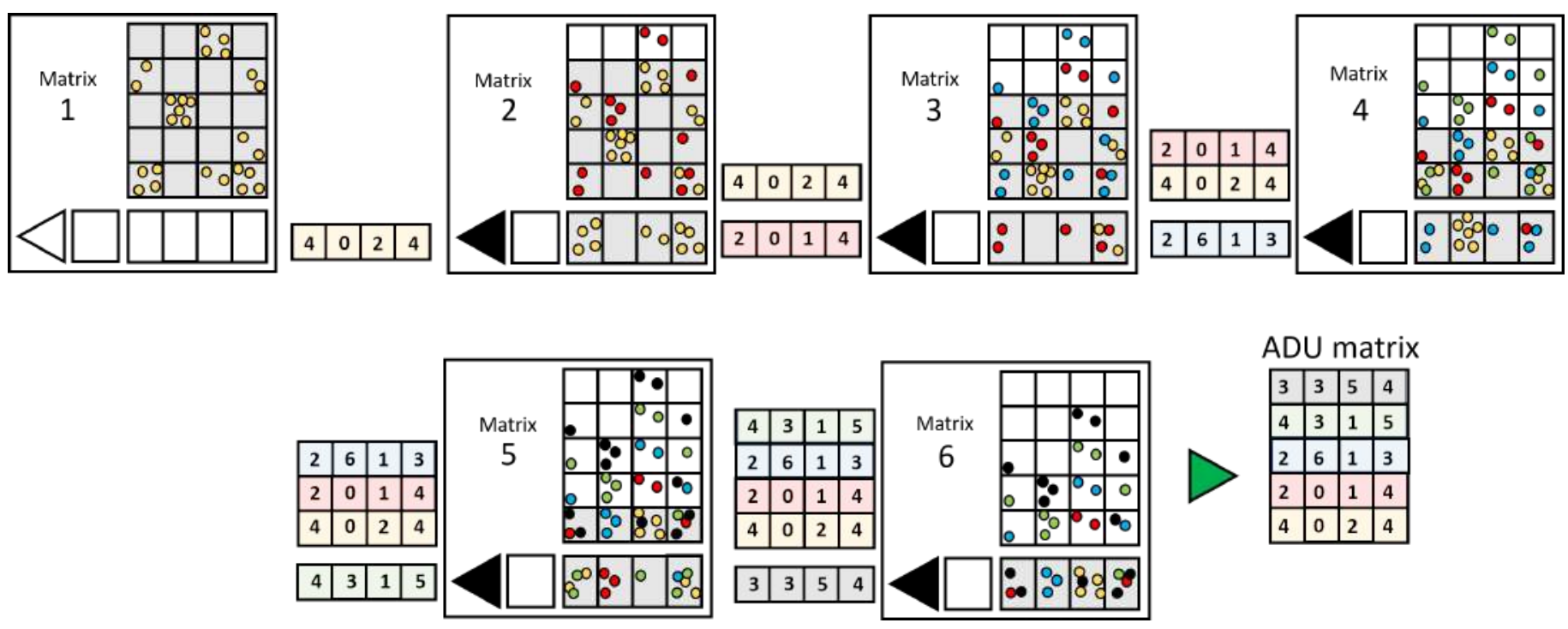

Figure 16. Sketch of the readout procedure, considering the RON contribution. The numbers in the matrix show the total loaded charge.

In Figure 15 the ADU matrix loaded by the CCD in the case of the RON was neglected is shown. However, considering the RON contributions, the ADU matrix drastically changes, as it is possible to see in the Figure 16.

In the case shown in Figure 16, it has been considered an impacting RON flux equal to half of the initial flux during the readout time of one row $\left(t_{R O}^{r}\right)$. This fraction $(0.5$, in this example) has been called $\beta$.

In more details, in Figure 16, the matrix 1 shows the initial photons flux (yellow dots) impacting in the CCD. During the first row readout time an additional contribution, due to the RON flux, impacts in the CCD (red dots in the matrix 2), and it is half of the initial photons number since we considered $\beta=0.5$. In the matrix 3 , another $\mathrm{RON}$ contribution (blue dots) impacts during the second row readout time. The same happens in the matrix 4 (green dots), and so on. Hence, the final ADU matrix (real case) dramatically differs from the one where the readout noise was neglected (ideal case).

The developed algorithm, once given the amount of time needed for information transfer between the rows and the standard acquisition time of the CCD, starts with identifying 
the amount of photons collected in a wrong position, considering the spatial distribution of the photons collected in the exposure time (step-1); once identified wrongly collected photons, the algorithm reallocates them in the right positions (step-2).

\subsubsection{Step-1: The Forward Method}

The first step of the algorithm (called Forward Method) consists in filtering out all the charges due to RON photons, which are pixel-position dependent. The algorithm works as shown in the example in Figure 17.

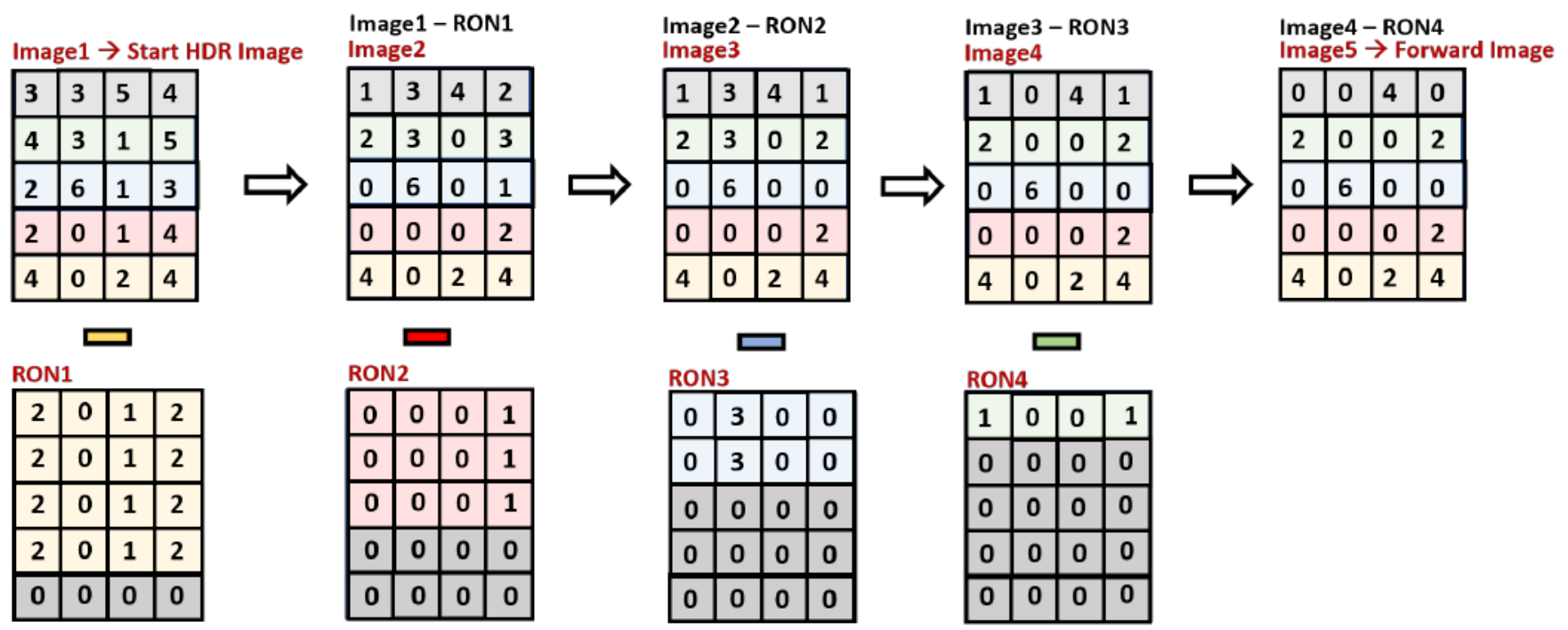

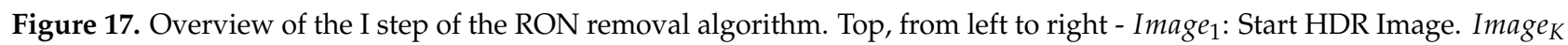
(with $\mathrm{K}$ from 2 to 5): Image $_{K-1}$ where all rows $\mathrm{j}$ (with $\mathrm{j} \geq \mathrm{K}$ ) have been subtracted by a contribution proportional (of a factor $\beta)$ to the loaded charge in the $(K-1)^{\text {th }}$ row of Image $_{K-1}$. In the last post-processed image, the Forward Image, the whole RON has been filter out. Bottom - matrix subtracted from the corresponding image on the top, at each step.

Considering the Start HDR Image, only the first row (yellow back-grounded) is unaffected by the RON, since it is read instantaneously. The second row (red back-grounded) contains both the charge due to photons impacting in it and the additional RON due to photons impacting during its $t_{R O}$, proportional to the flux that can be estimated by the previous rows (only the first one, in this case). The third row (blue back-grounded) contains the charges due to photons impacting on it plus two additional RON contributions, proportional to the fluxes read in the previous two rows, and so on. The algorithm, starting from the Start HDR image, subtracts step-by-step the RON contribution in each row loaded during the $t_{R O}$.

Starting from the Start HDR Image (the $1^{\text {st }}$ image), 1023 images have been analyzed stepby-step: the $K^{\text {th }}$ image (with $\left.K=2 \rightarrow 1024\right)$ corresponds to the $(K-1)^{\text {th }}$ image in which all rows $\mathrm{j}$ (with $\mathrm{j} \geq \mathrm{K}$ ) have been subtracted by a contribution proportional (of a factor $\beta$ ) to the loaded charge in the $(K-1)^{\text {th }}$ row of the $\operatorname{Image}_{K-1}$. In the final post-processed image, called Forward Image, all RON contributions have been filter out. The Forward Image is characterized by the same ADUs of the image obtained neglecting the RON contribution (Figure 15), demonstrating the effectiveness of the described procedure.

Moreover, considering the difference from the Start HDR Image and the Forward Image, it is possible to obtain the so called "Displaced RON Image" consisting of all RON photons collected during the RO time and placed in wrong positions (see Figure 18).

These RON photons from one hand represent a spurious contribution because they are placed in wrong pixel position but, on the other hand, they are "good" events because are true photons coming from the plasma setup that impact on the CCD. It has been possible to relocate them in the correct pixel position, increasing the statistics. 


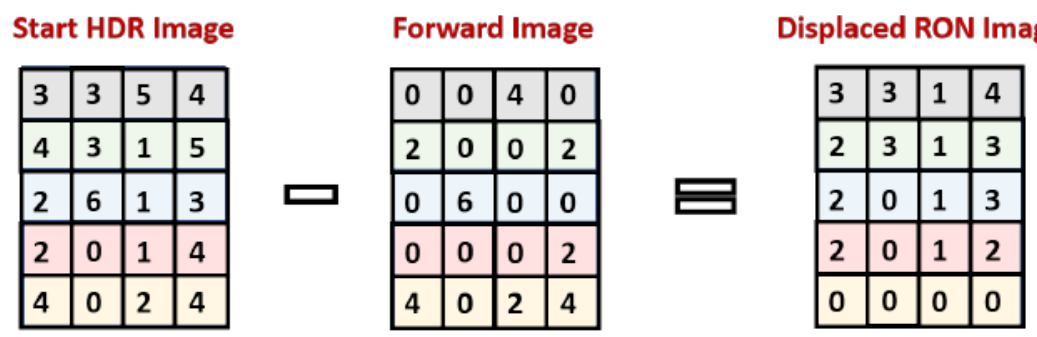

Figure 18. From left to right: Start HDR Image; Forward Image; Displaced Readout Noise Image.

In the previous example, a $\beta$ parameter of 0.5 has been considered, but the real experimental $\beta$ parameter (which depends of the $t_{R O}$ ) has been estimated. The measurements have been performed setting a readout frequency of $1 \mathrm{MHz}\left(10^{6}\right.$ pixels / sec), so the readout time of one single pixel $t_{R O}^{p}$ is $10^{-6} \mathrm{~s}$; subsequently, the readout time of one row $R O_{t}^{r}$ results $1024 \cdot 10^{-6}=0.0010124 \mathrm{~s}$. Therefore, the $\beta$ parameter is:

$$
\beta=\frac{t_{R O}^{p}}{t_{\text {exp }}}
$$

$\beta$ represents, in other words, the fraction of flux impacting in a single row during its own $t_{R O}$. For $I_{\text {Plasma }}$, where $t_{\text {exp }}=0.5 \mathrm{~s}, \beta_{I_{\text {Plasma }}}=0.002$; whilst for $I_{\text {Pole }}$, where $t_{\exp }=0.05 \mathrm{~s}$, $\beta_{I_{\text {Pole }}}=0.02$.

The measured Start HDR, Forward and Displaced RON images are shown in Figure 19a, 19b and $19 \mathrm{c}$ respectively for Ar-, Ti- and Ta-energy filtering.
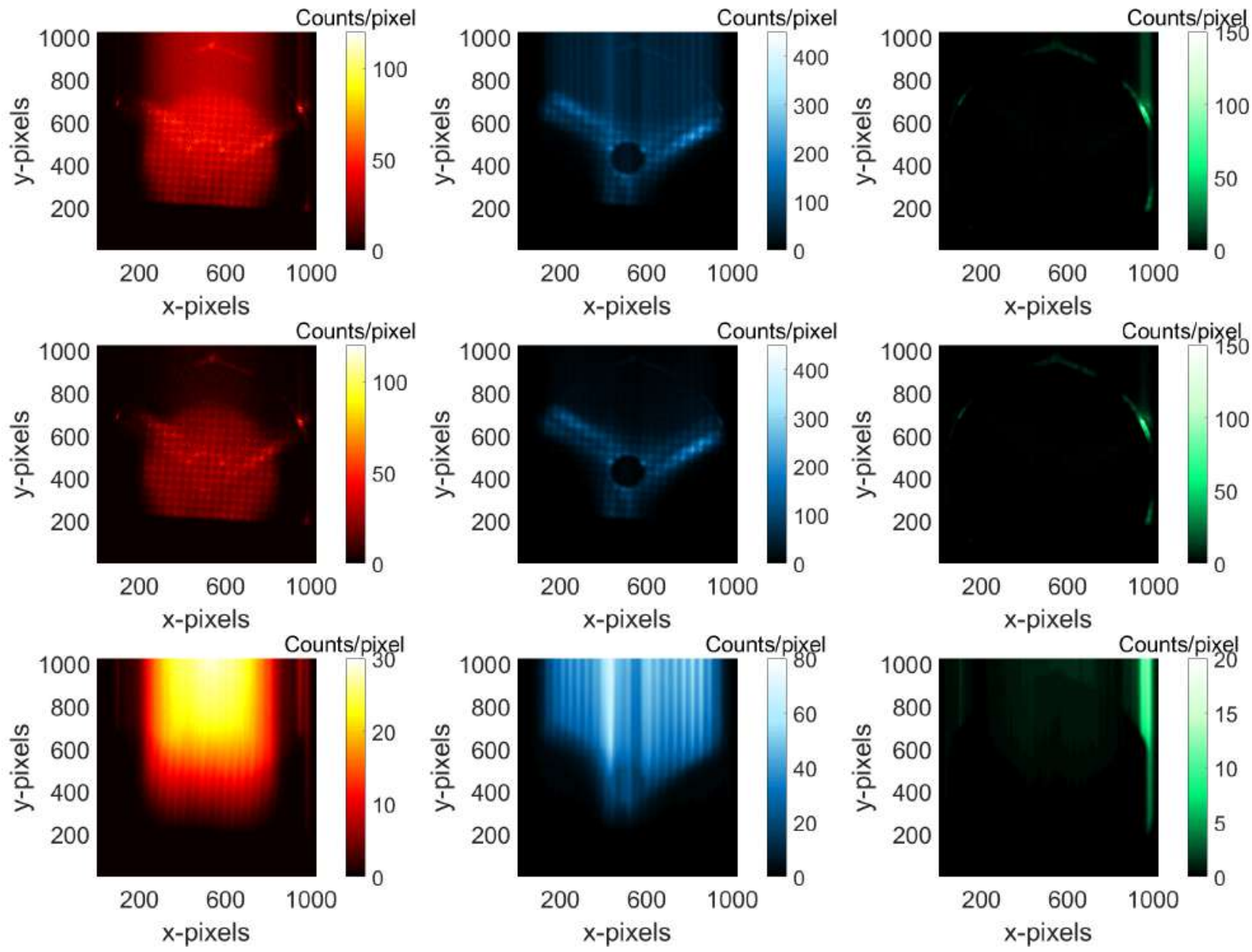

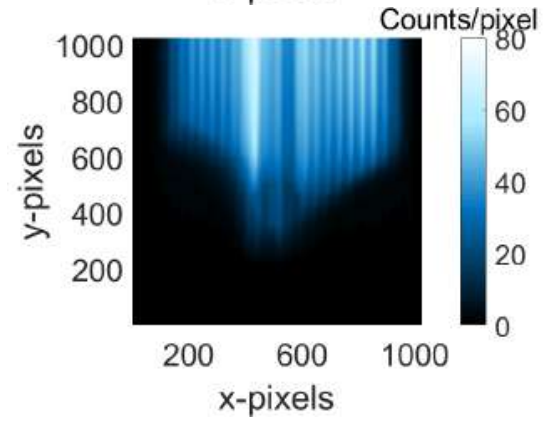

(b) Ti-energy filtered images

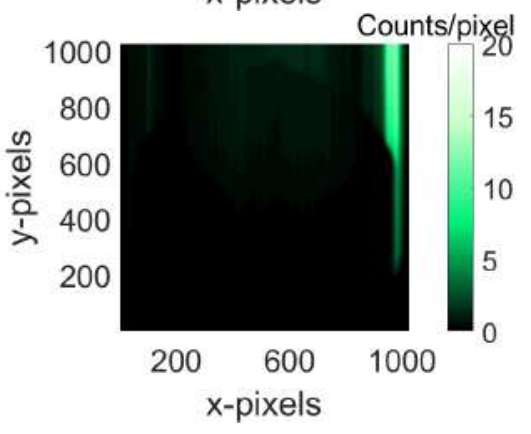

(c) Ta-energy filtered images

(a) Ar-energy filtered images

Figure 19. Top: Start HDR images. Middle: Forward images. Bottom: Displaced RON images 


\subsubsection{Step: The Backward Method}

The genuine events shown in the Displaced RON Images (Figure 19, bottom row) must be "relocated" in the correct position by means of the second step.

Thus, a new matrix called Weight Matrix WM - obtained multiplying the Forward Image by $\beta$ - was defined. A sub-matrix $W_{i}$ was associated to each $i^{t h}$ row, normalized and expressed in percentage (with nonzero elements only in rows $\mathrm{j} \geq \mathrm{i}$ ). The elements of the $W_{i}$ matrices give the pixel-position dependent weight $w_{k, j}$ of the RON coming from each pixel during the readout time.

The second step of the algorithm consists of redistributing the charge loaded in each pixel $(\mathrm{i}, \mathrm{j})$ of the Displaced RON Image, in all the pixels $(\mathrm{k}, \mathrm{j})$, with $\mathrm{k}=(\mathrm{i}+1) \rightarrow 1024$, of a new matrix called $R O N_{i}$ by means of the weights $w_{k, j}$. The $W_{i}$ and $R O N_{i}$ are shown in Figure 20, for $\mathrm{i}=1$ to 4 .

The sum of all the $R O N_{i}$ matrices gives the so called "Placed Readout-Noise Image", shown in Figure 21, that corresponds to the Displaced RON Image where all charge has been redistributed in the correct pixel-position. It is possible, in fact, to verify that the total charge is the same: total ADU $=31$ both in Placed RON Image and in Displaced RON Image (see also the Table 3 for comparing ADU of the experimental acquired images).

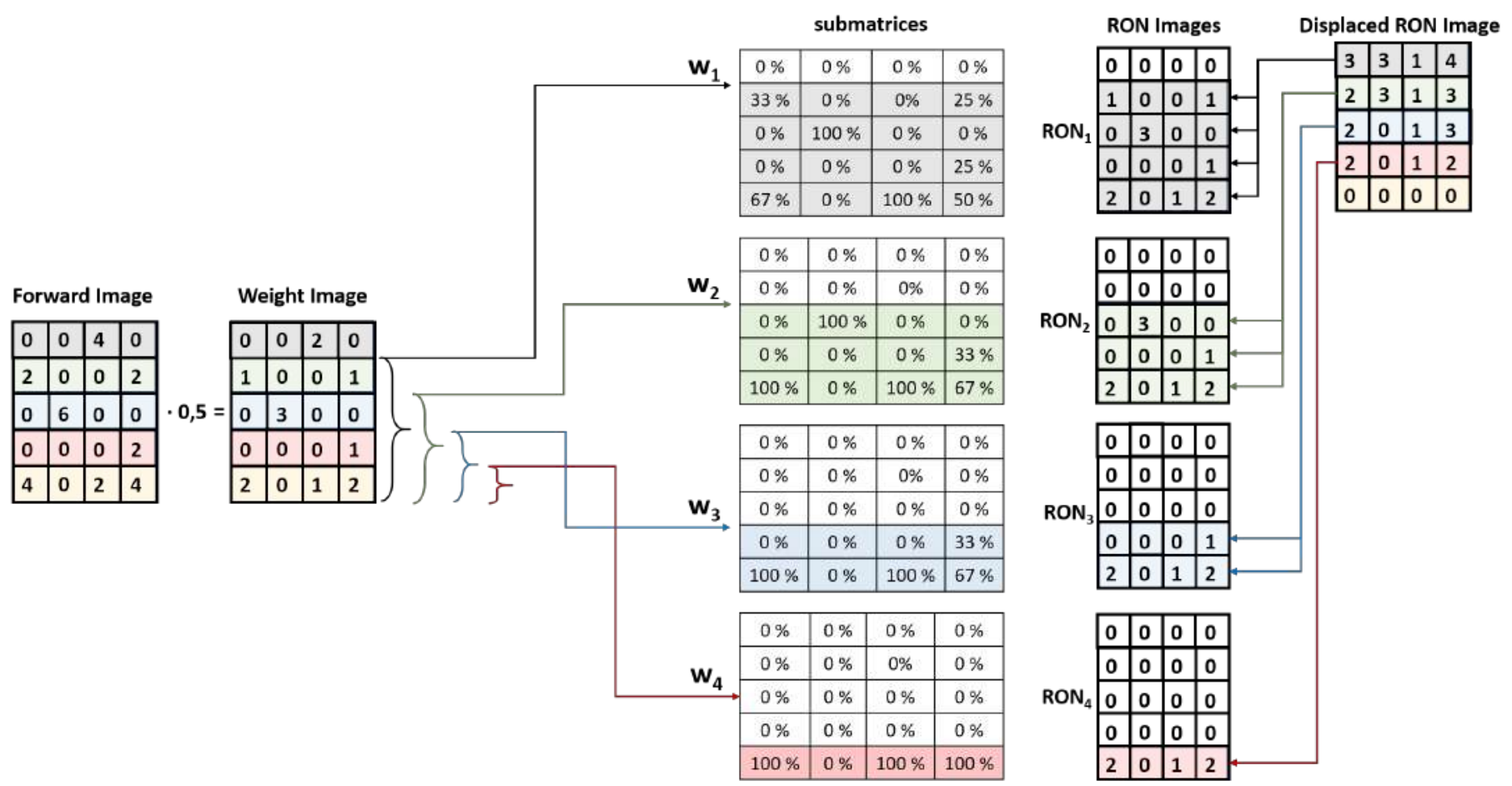

Figure 20. From left to right: Forward Image. Weighting Matrix WM. Sub-matrices $W_{i}$ having $\mathrm{j} \geq \mathrm{i}$ associate at each $\mathrm{WM} i{ }^{\text {th }}$ row. $R O N_{i}$ matrices obtained stating from the Displaced $R O N$ Image by means of weights $w_{k, j}$, elements of the $W_{i}$ matrices for each row $i$. Displaced RON Image.

Placed RON Image
$\left.\left.\begin{array}{|l|l|l|l|l|}\hline 0 & 0 & 0 & 0 \\
\hline 1 & 0 & 0 & 1 \\
\hline 0 & 6 & 0 & 0 \\
\hline 0 & 0 & 0 & 3 \\
\hline 8 & 0 & 4 & 8 \\
\hline 1 & 0 & 0 & 0 & 0 \\
\hline 0 & 3 & 0 & 0 \\
\hline 0 & 0 & 0 & 1 \\
\hline 2 & 0 & 1 & 2 \\
\hline\end{array}\right\} \begin{array}{|l|l|l|l|}\hline 0 & 0 & 0 & 0 \\
\hline 0 & 0 & 0 & 0 \\
\hline 0 & 3 & 0 & 0 \\
\hline 0 & 0 & 0 & 1 \\
\hline 2 & 0 & 1 & 2 \\
\hline\end{array}\right\}$\begin{tabular}{|l|l|l|l|}
\hline 0 & 0 & 0 & 0 \\
\hline 0 & 0 & 0 & 0 \\
\hline 0 & 0 & 0 & 0 \\
\hline 0 & 0 & 0 & 1 \\
\hline 2 & 0 & 1 & 2 \\
\hline 2 & 0 & 1 & 2 \\
\hline
\end{tabular}

Figure 21. Placed RON Image obtained by the sum of all the $R O N_{i}$. 
Even if the Forward Matrix can be used to calculate the Weight Matrix, as shown in the previous example, it is possible to demonstrate that there is an improvement when replacing it with the one obtained applying in the Forward Matrix a pixel-position dependent threshold. The reason is due to the fact that there is the background (BGN) contribution during $\mathrm{RO}$ acquisition as well.

To this scope, a pixel-position dependent threshold has been defined and applied in the Forward Matrix:

- first, 200 image-frames acquired setting the lowest $t_{\exp }$ possible in the driver $\left(t_{\exp }=\right.$ $20 \mu s$ ) have been used to estimate the BGN threshold. The sum of the 200 event-counted frames is shown in Figure 22a, it has been normalized for the frames number and the $\sigma$ level, pixel-by-pixel, has been considered as BGN threshold (Figure 22b).

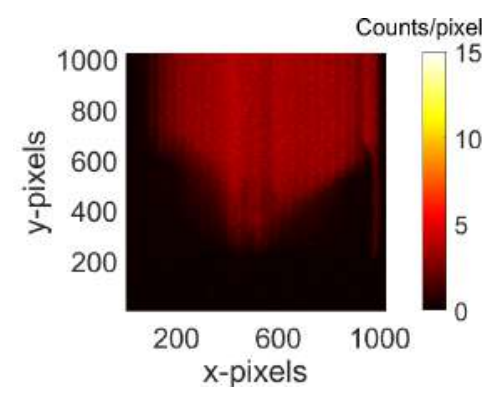

(a)

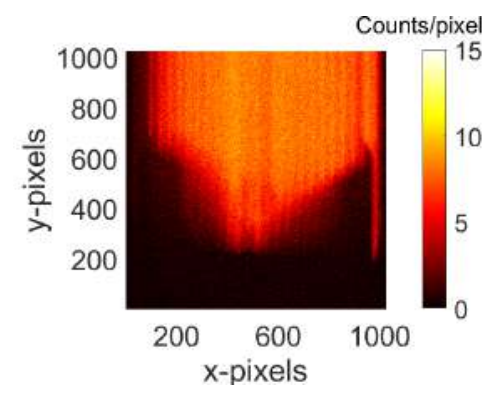

(b)

Figure 22. a) Background Image. b) Threshold Image.

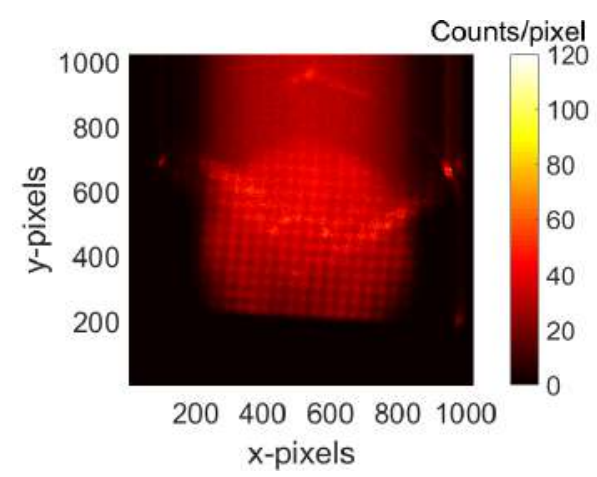

(a) Start HDR Image

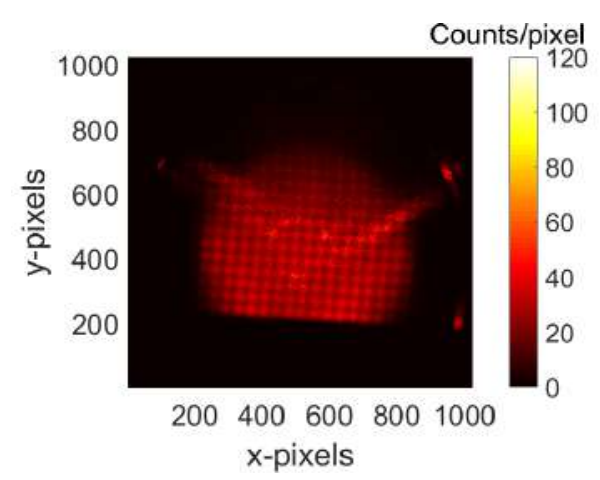

(d) Placed RON Image

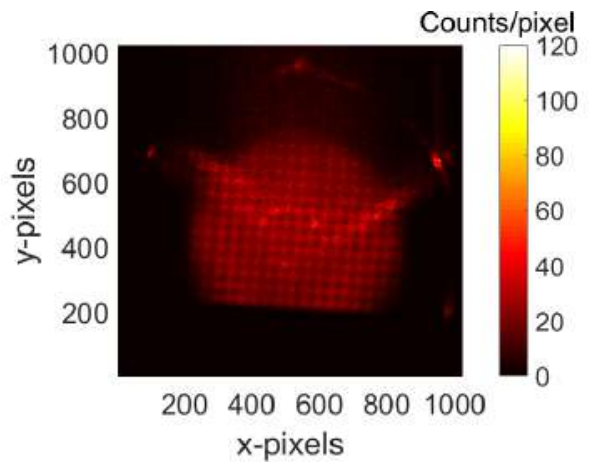

(b) Forward Image

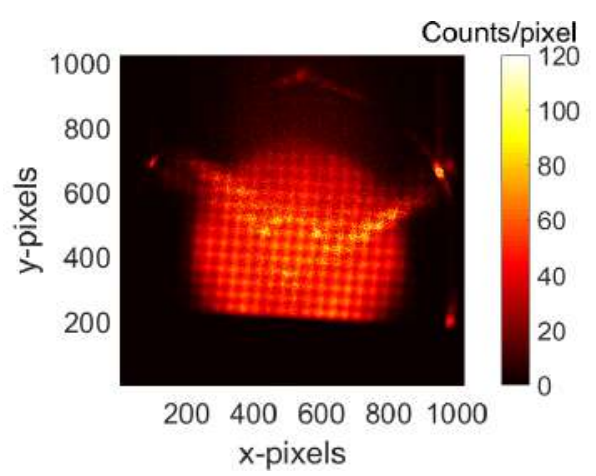

(e) Backward Image

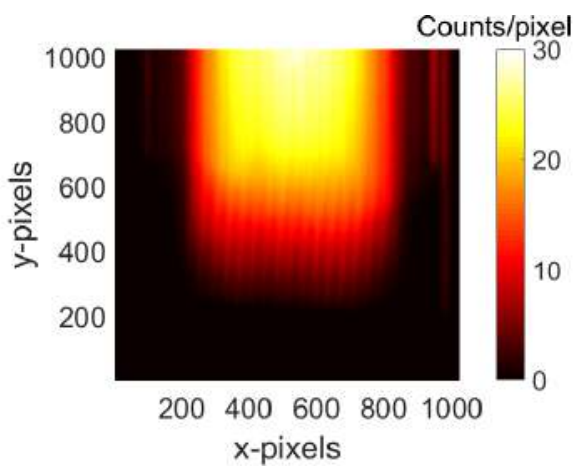

(c) Displaced RON Image

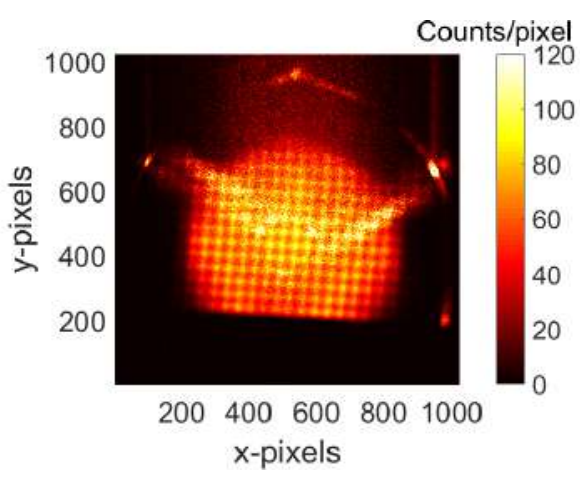

(f) Normalized Backward Image

Figure 23. Sequence of the (Argon-energy filtered) images obtained during the data-processing by the RON-r algorithm.

- The Start HDR, Forward and Displaced RON images are shown in Figure 23a, 23b and 23 c respectively. 
- $\quad$ Setting the BGN threshold in the Forward Image, the Weight Image was obtained and used to estimate the Placed RON Image (Figure 23d). It contains the same RON charge collected in the Displaced RON image, but placed in the correct positions.

- $\quad$ The sum of the Forward Image and of the Placed RON Image gives the so called "Backward Image", shown in Figure 23e, which contains the whole charges (collected during the measurement time $+\mathrm{RON}$ time) in the correct pixel-positions.

- For the data collected during the $\mathrm{RO}$, the effective $t_{\text {exp }}$ is pixel-position dependent, therefore the Backward Image has been also properly time-normalized. The final postprocessed image is called "Normalized Backward Image" and is shown in the Figure $23 f$.

The images shown in Figure 23 are Argon-energy filtered.

It is worth to point put how the post-processed images quality dramatically improves if compared to the original one. The proper handling of RON after the elaboration of the "Normalized Backward Image" is evident, and also spatial resolution increases.

In Table 3 it is possible to observe that the total counts of the Start HDR Image (a) are the same of the ones of the Backward Image (e): the only difference is that all the RON photons have been relocated in the correct positions. The same observation can be done for the Displaced RON Image (c) and the Placed RON Image (d). Of course, the sum of the total counts of the Forward Image (b) and the Displaced RON Image (c) is equal to the one in the Start HDR Image (a). The slight difference between the counts of the Weighing Image (i) and the Forward Image (b) is due to the events filtering after the elimination of the BGN.

Table 3. Total counts of each image (Argon-energy filtered) used in the RON-r algorithm.

\begin{tabular}{|c|c|c|}
\hline & Name and description of the Image & $\begin{array}{l}\text { Total } \\
\text { Counts }\end{array}$ \\
\hline a) & $\begin{array}{l}\text { Start HDR Image: } \\
\text { HDR Image with Readout Noise }\end{array}$ & $1.49 \cdot 10^{7}$ \\
\hline b) & $\begin{array}{l}\text { Forward Image: } \\
\text { Original Image without the Readout Noise removed by means of the forward method }\end{array}$ & $0.74 \cdot 10^{7}$ \\
\hline c) & $\begin{array}{l}\text { Displaced Readout-Noise Image: } \\
\text { Subtraction between the Original Image and the Forward Image }\end{array}$ & $0.75 \cdot 10^{7}$ \\
\hline d) & $\begin{array}{l}\text { Placed Readout-Noise Image: } \\
\text { Displaced Readout-Noise Image with photons placed in the correct position by the backward method }\end{array}$ & $0.75 \cdot 10^{7}$ \\
\hline e) & $\begin{array}{l}\text { Backward Image: } \\
\text { Sum pixel-by-pixel of the Forward Image and the Placed Readout-Noise Image }\end{array}$ & $1.49 \cdot 10^{7}$ \\
\hline f) & $\begin{array}{l}\text { Normalized Backward Image: } \\
\text { Time normalized Backward Image }\end{array}$ & $2.27 \cdot 10^{7}$ \\
\hline g) & $\begin{array}{l}\text { Background Image: } \\
\text { Mean image of } 200 \text { image-frames acquired setting an exposure time } \sim \text { readout time }\end{array}$ & $0.16 \cdot 10^{7}$ \\
\hline h) & $\begin{array}{l}\text { Threshold Image: } \\
\text { Square root of the Background (and Normalized) Image pixel-by-pixel }\end{array}$ & $0.42 \cdot 10^{7}$ \\
\hline i) & $\begin{array}{l}\text { Weighing Image: } \\
\text { Forward Image filter out all events lower than the threshold pixel-by-pixel taken in the Threshold Image }\end{array}$ & $0.72 \cdot 10^{7}$ \\
\hline
\end{tabular}

In Figure 24 the signal-to-noise ratio after the application of the three different $R O N-r$ methods is reported, pointing out the comparatively huge improvement (a factor of 30, if compared to the original image) obtained after the implementation of the whole algorithms sequence. 


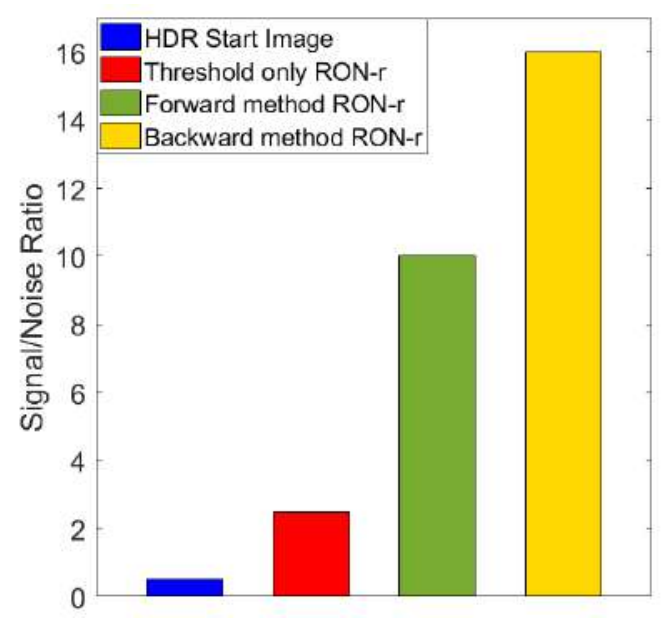

Figure 24. Increase of the Signal/Noise Ratio after applying the sequence of RON-r methods, compared to the Original Image.

$364 \quad$ The corresponding $\mathrm{Ti}$ and Ta-energy filtered images are shown in the Figures 25 and 36526 , respectively.

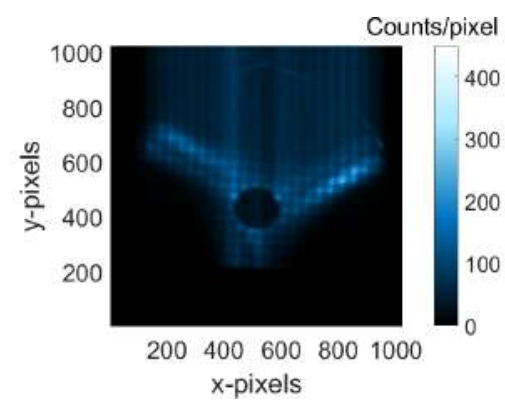

(a) Start HDR Image

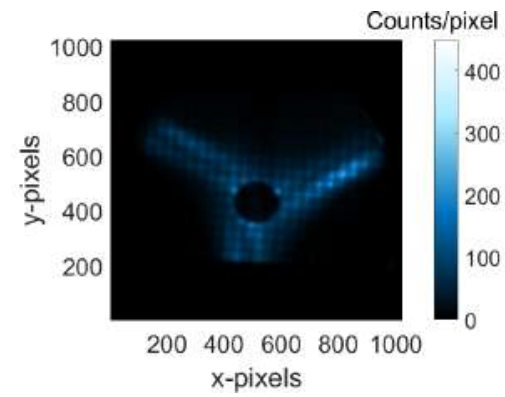

(d) Placed RON Image

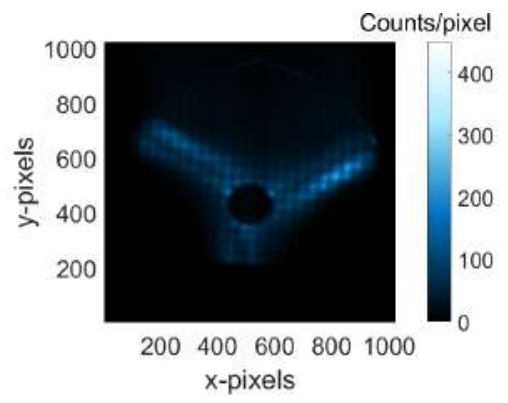

(b) Forward Image

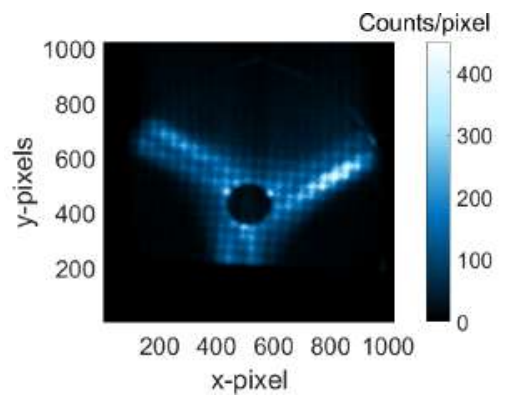

(e) Backward Image

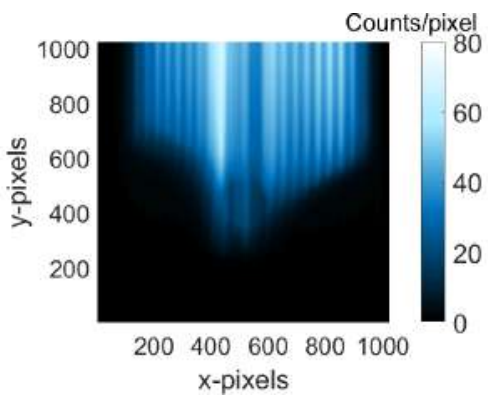

(c) Displaced RON Image

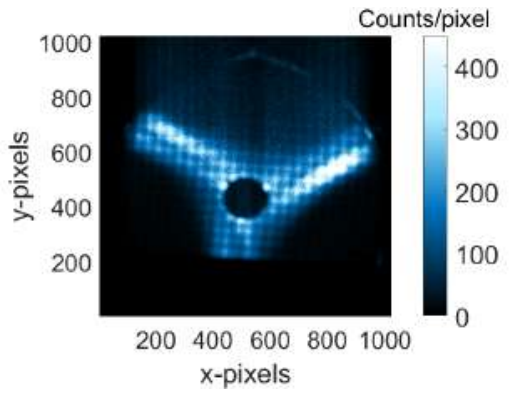

(f) Normalized Backward Image

Figure 25. Sequence of the (Titanium-energy filtered) images obtained during the data-processing by the RON-r algorithm. 


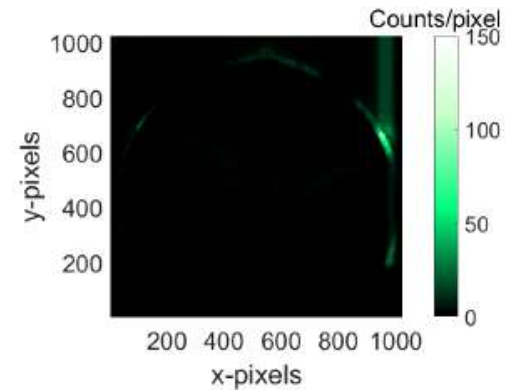

(a) Start HDR Image

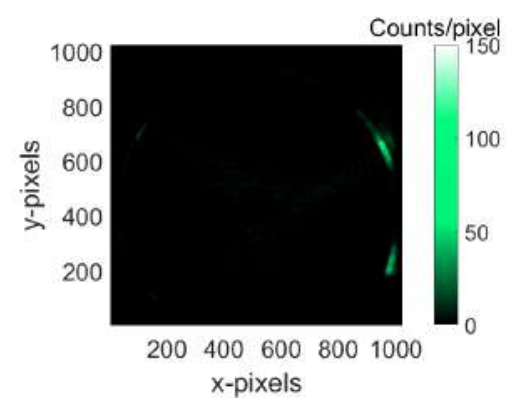

(d) Placed RON Image

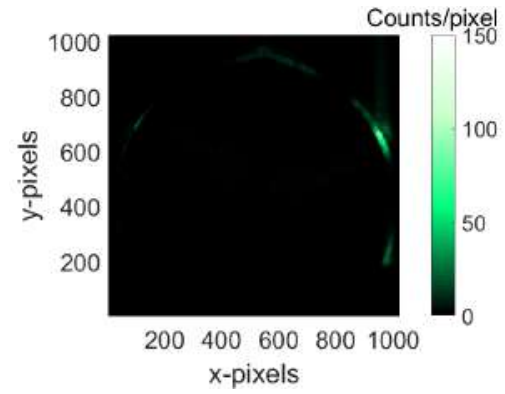

(b) Forward Image

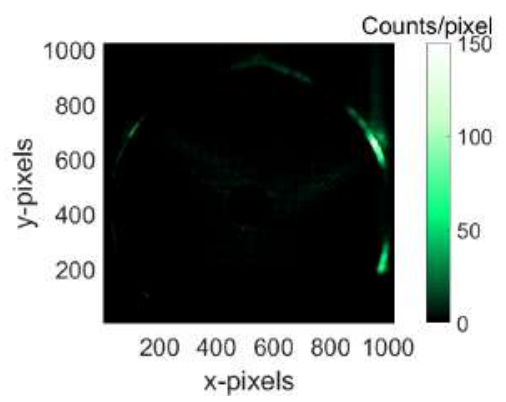

(e) Backward Image

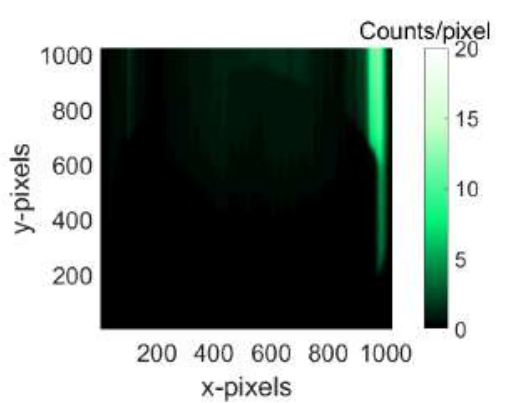

(c) Displaced RON Image

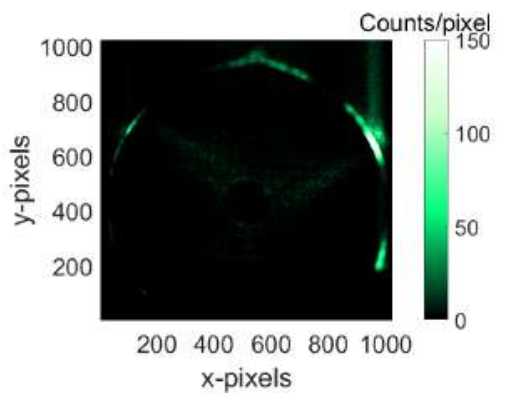

(f) Normalized Backward Image

Figure 26. Sequence of the (Tantalum-energy filtered) images obtained during the data-processing by the RON-r algorithm.

Finally, in the Figures 27 the comparison between the $I_{\text {Plasma }}$ images, the HDR postprocessed images with RON and the Readout Noise Removed post-processed images is shown (note that the full scale is different in the $I_{\text {Plasma }}$ images compared to the other ones). Event counted energy-unfiltered images (27a), Ar-energy filtered images (27b), Ti-energy filtered images (27c) and Ta-energy filtered images (27d) are shown.

It is possible to observe the improvement of the final post-processed images, where the "strips" due to RON completely disappeared. First plasma proprieties investigations performed by applying the whole set of aforedescribed algorithms is reported in [38]. 

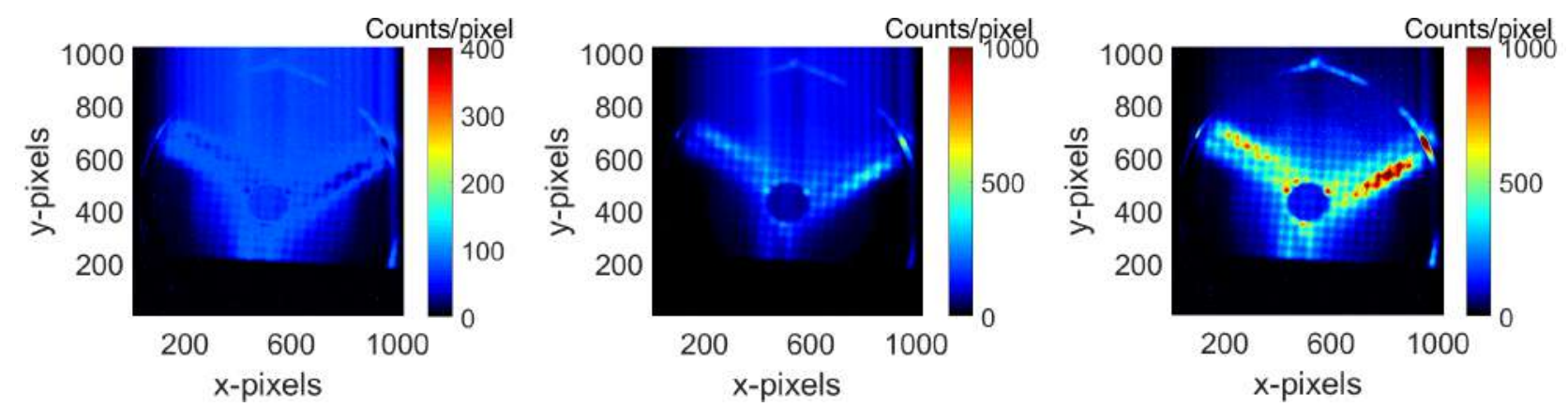

(a)
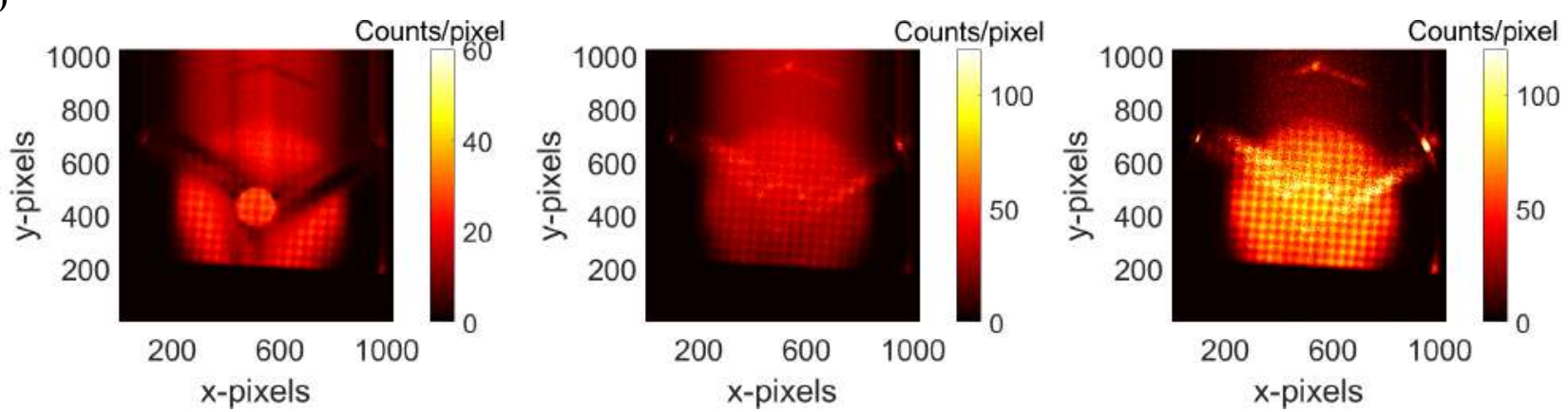

(b)
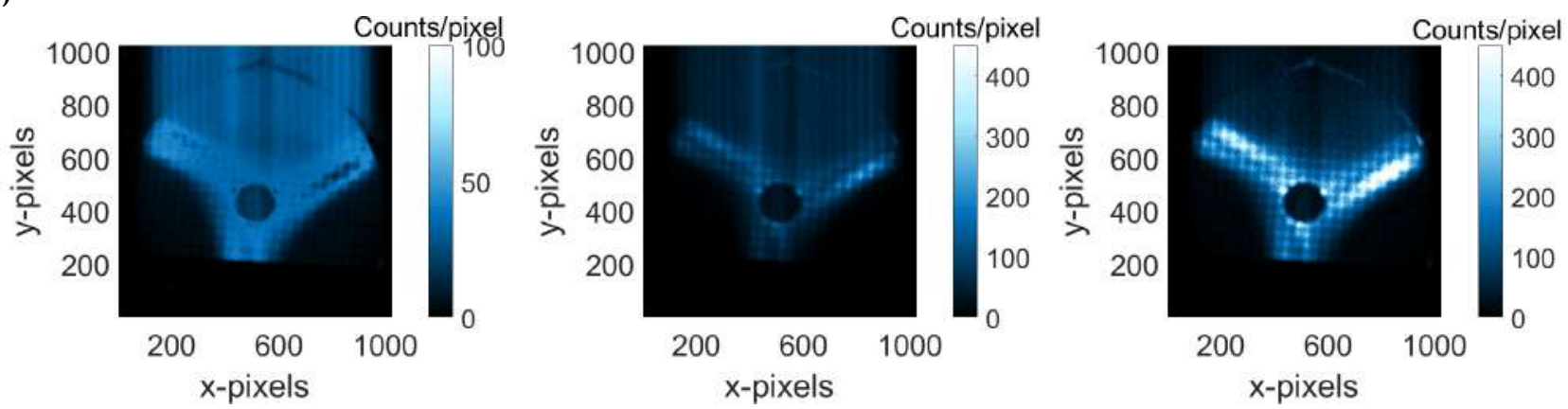

(c)
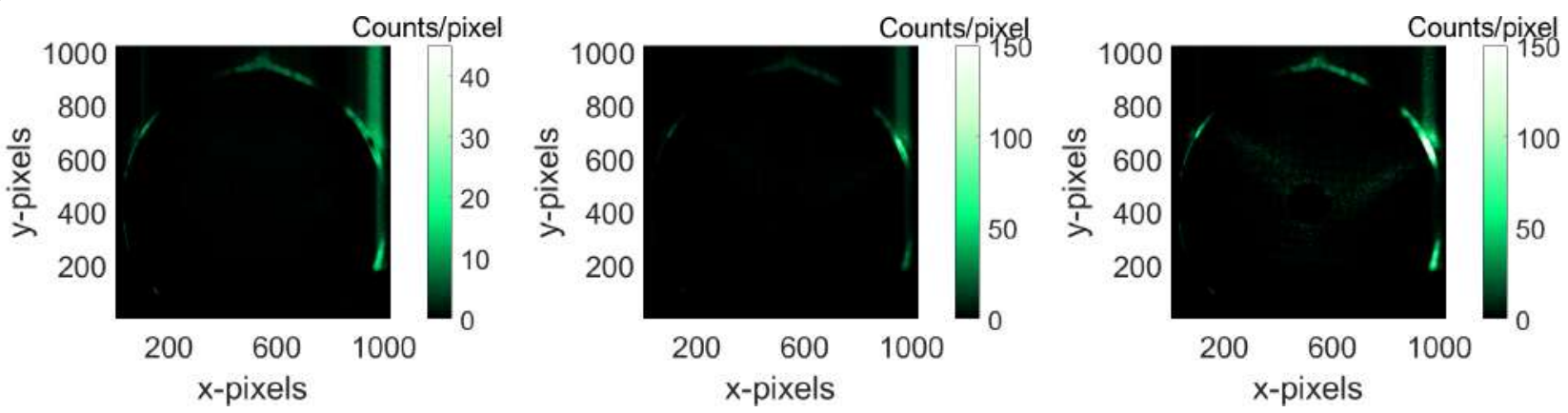

(d)

Figure 27. First column: the $I_{\text {Plasma }}$ grouped image. Second column: the HDR post-processed image. Third column: the Readout Noise Removed post-processed image. a) Event counted energy-unfiltered image. b) Ar-energy filtered image. c) Ti-energy filtered image. d) Ta-energy filtered image. (Note the different the full scale in the first row).

Error analysis of the number of counts in each pixel of the single photon countedimage has been estimated by the square-root of counts. The Figure 28 illustrates, for each pixel, the relative error $\sigma_{r e l}$ of the counts for the event counted Ar-filtered image (28a) and for the event counted Ti-filtered image (28b). The relative errors are $<10 \div 15 \%$ in the plasmoid and lower than $<4 \div 8 \%$ in the magnetic branches regions. In the magnetic poles the relative errors are around $10 \%$. These are the typical uncertainties of the number of counts in each pixel, which affect the performed measurements. These numbers are 
corresponding to single pixels. We may note here that due to the high resolution we can easily (without remarkable lost of the spatial resolution) increase the statistics and consequently decrease the uncertainty by summing up a group pixels corresponding to a given ROI.

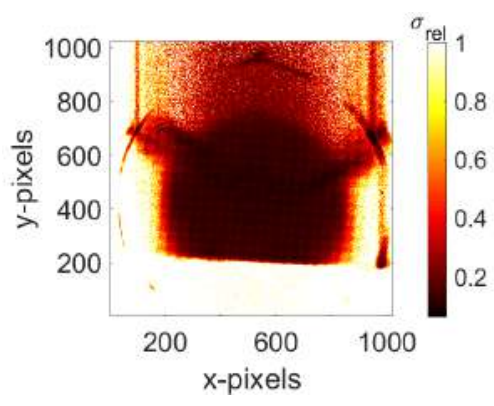

(a)

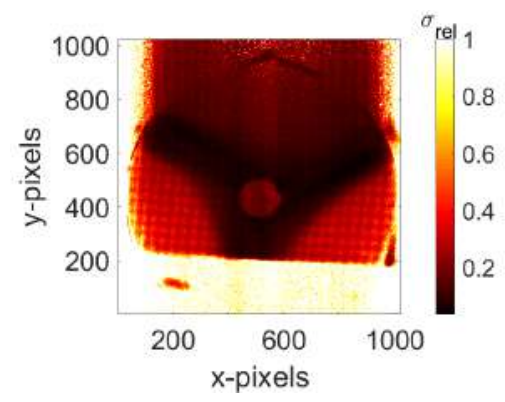

(b)

Figure 28. The images show the errors of the number of counts in each pixel: a) Ar-filtered image; b) Ti-filtered image.

\section{Plasma Analysis: a Short Overview}

Once post-processed the X-ray images by means of the advanced algorithm, two important complementary analyses of the SPhC images can be simultaneously performed: a) the HDR Energy-filtered Imaging and b) the HDR Space-filtered Spectroscopy.

By means of the HDR energy-filtered imaging it is possible to obtain the imaging of the elemental distribution, distinguishing the emission, and the correspondent image, coming from each material. Pixels highlighting photons due to fluorescence peaks are shown in the last column of Figure 27: 27b, 27c, 27d respectively for the Ar-, Ti- and Ta-energy-filtered. In this way, it is possible to study the plasma structure and morphology changes and to investigate in details the plasma confinement and losses dynamics.

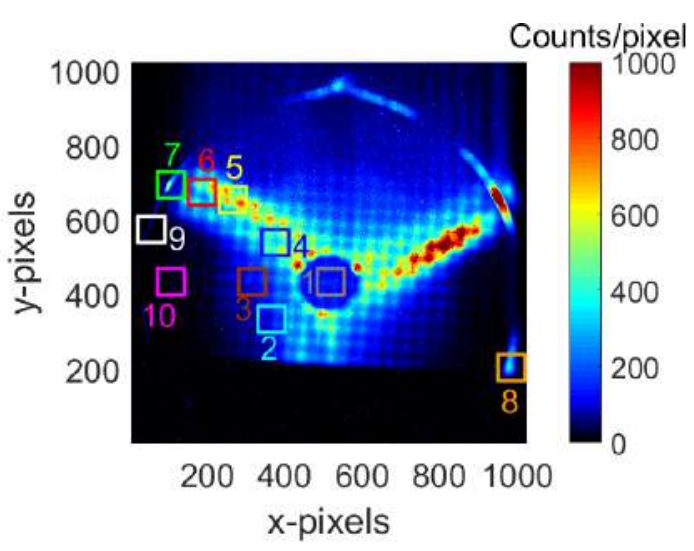

(a)

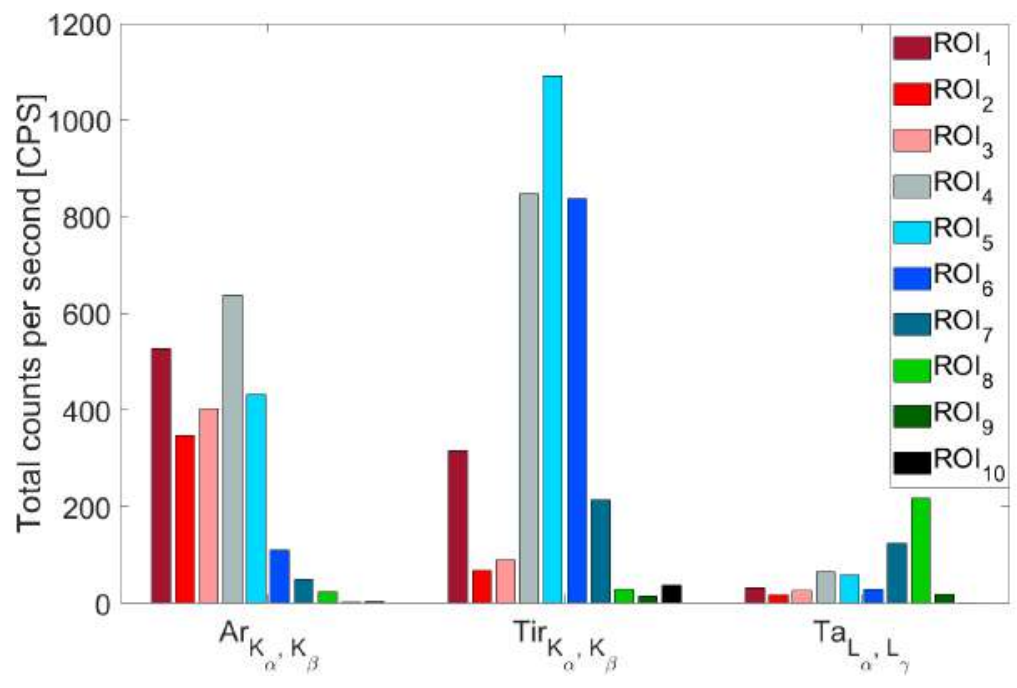

(b)

Figure 29. (a) Event-counted and not-energy-filtered HDR image, with ten highlighted ROIs. (b) Histogram of the emission contribution (expressed in total counts per second) from each ROI, for each fluorescence line $\left(A r_{K_{\alpha}, K \beta}, T i_{K_{\alpha}, K \beta}, T a_{L_{\alpha}, L \gamma}\right)$.

A complementary analysis consists in the selection of a ROI in the not-energy filtered HDR image, then investigating the spectral composition of that part only. This kind of space-filtered spectroscopy enables a quantitative study about X-ray emission proprieties by plasma electrons or deconfined electrons hitting on the chamber walls. An example of 10 selected ROIs is reported in Figure 29a.

A quantitative estimation of the total counts per second emitted in each energy interval 
of interest has been performed by means of fitting the main peaks of Argon, Titanium and Tantalum $\left(A r_{K_{\alpha}, K \beta}, T i_{K_{\alpha}, K \beta}, T a_{L_{\alpha}, L \gamma}\right)$ in the spectrum. The results are summarized in Figure 29b. The histogram shows, for each fluorescence line reported in the horizontal axis, the contribution coming from each ROI (colour of the histogram), i.e. the elemental composition. These contributions are expressed in CPS. It is possible to note that the plasma emission comes from both internal and external regions to the ECR surface (ROIs 1, 2, 3 and 4 are inside the ECR layer, while ROIs 5 and 6 are placed outside). This put in evidence the deconfined plasma flowing along the magnetic branches until the more external region $\mathrm{ROI}_{6}$. More details about the experimental results are described in $[30,38]$.

Finally, it is important to mention that both the whole spectrum and the spectra in each ROI can be analysed in order to locally estimate the plasma parameters, in term of electron temperature and electron and ion densities, by comparing experimental spectrum vs. theoretical one. Recently, a novel numerical tool for analyzing spatially anisotropic electron populations in ECR plasmas has been developed [39] at INFN-LNS. In perspective, the plasma emitted spectrum can be reelaborated in order to measure the so-called spectral emissivity curve providing the total equivalent number of photons emitted in the given energy interval over the entire sold angle measured by the detector. Typically, for a precise detector's solid angle estimation dedicated Monte Carlo simulations needs to be implemented.

\section{Conclusions}

In this paper an innovative analytical method developed for performing spaceresolved high resolution $\mathrm{X}$-ray spectroscopy, has been presented. The algorithm has been finalized to analyze the raw data acquired using the X-ray pin-hole camera tool and represents a powerful method for plasma structure evaluation, plasma confinement and losses dynamics investigations and for locally determining plasma density and temperature. In particular, the developed tools now enable Single Photon-Counting operations minimizing read-out effects and with high signal-to-noise rations, allowing energy-resolved investigation pixel-by-pixel, also providing the local plasma emitted spectrum in a HighDynamic-Range (HDR) mode. The experimental setup is now going to be updated in order to allow simultaneously space and time-resolved plasma spectroscopy during transients, stable and turbulent regimes.

Author Contributions: Conceptualization, Eugenia Naselli, Richard Rácz, Sandor Biri, Giuseppe Torrisi, Alessio Galatà and David Mascali; Data curation, Eugenia Naselli, Richard Rácz, Sandor Biri, Maria Mazzaglia, Alessio Galatà and David Mascali; Formal analysis, Eugenia Naselli, Richard Rácz, Sandor Biri and David Mascali; Funding acquisition, Sandor Biri, Alessio Galatà and David Mascali; Investigation, Eugenia Naselli, Richard Rácz, Sandor Biri, Maria Mazzaglia, Zoltan Perduk, Alessio Galatà and David Mascali; Methodology, Eugenia Naselli, Richard Rácz, Sandor Biri and David Mascali; Project administration, Sandor Biri and David Mascali; Resources, Eugenia Naselli, Richard Rácz, Sandor Biri, Maria Mazzaglia, Luigi Celona, Santo Gammino, Giuseppe Torrisi, Zoltan Perduk, Alessio Galatà and David Mascali; Software, Eugenia Naselli, Richard Rácz, Sandor Biri and David Mascali; Supervision, Sandor Biri and David Mascali; Visualization, Eugenia Naselli, Richard Rácz, Sandor Biri, Maria Mazzaglia, Luigi Celona, Santo Gammino, Giuseppe Torrisi, Zoltan Perduk, Alessio Galatà and David Mascali; Writing - original draft, Eugenia Naselli; Writing - review \& editing, Eugenia Naselli, Richard Rácz, Sandor Biri, Maria Mazzaglia, Luigi Celona, Santo Gammino, Giuseppe Torrisi, Zoltan Perduk, Alessio Galatà and David Mascali.

Acknowledgments: The authors gratefully acknowledge the support of INFN by the Grants PANDORA (5th Nat. Comm.) and PANDORA_Gr3 (3rd Nat. Comm.).

Conflicts of Interest: The authors declare no conflict of interest.

\section{References}

1. Naselli, E.; Mascali, D.; Biri, S. et al. Multidiagnostics setups for magnetoplasmas devoted to astrophysics and nuclear astrophysics research in compact traps. JINST 2019, 14, C10008. 
2. Biri, S.; Pálinkás, J.; Perduk, Z. et al. Multi diagnostic setup at the Atomki-ECRIS to investigate the two-close-frequency heating phenomena. JINST 2018, 13, C11016.

3. Mascali, D.; Musumarra, A.; Leone, F. et al. PANDORA, a new facility for interdisciplinary in-plasma physics. Eur. Phys. J. A 2017, 53, 145.

4. Naselli, E.; Mascali, D.; Caliri, C. et al. Nuclear $\beta$-decays in plasmas: how to correlate plasma density and temperature to the activity. EPJ Web of conference 2020, 227, 02006.

5. Mascali, D.; Busso, M.; Mengoni, A. et al. The PANDORA project: an experimental setup for measuring in-plasma $\beta$-decays of astrophysical interest. EPJ Web of conference 2020, 227, 01013.

6. Nebem, D.; Fogleman, J.; Isherwood, B. et al. X-ray investigation on the Superconducting Source for Ions (SuSI). JINST 2019, 14, C02008.

7. Li, J.B.; Li, L.X.; Li, L.B. et al. Influence of electron cyclotron resonance ion source parameters on high energy electrons. Rev. Sci. Instrum. 2020, 91, 083302.

8. Benitez, J.; Lineis, C.; Phair, L. et al. Dependence of the Bremsstrahlung Spectral Temperature in Minimum-B Electron Cyclotron Resonance Ion Sources. IEEE Trans. Plasma Sci. 2017, 45, 7.

9. Isherwood, B.; Machicoane, G. Measurement of the energy distribution of electrons escaping confinement from an electron cyclotron resonance ion source. Rev. Sci. Instrum. 2020, 91, 025104.

10. Ropponen, T.; Tarvainen, O.; Toivanen, V. et al. The effect of rf pulse pattern on bremsstrahlung and ion current time evolution of an ECRIS. Rev. Sci. Instrum. 2010, 81, $02 \mathrm{~A} 302$.

11. Mascali, D.; Celona, L.; Maimone, F. et al. X-ray spectroscopy of warm and hot electron components in the CAPRICE source plasma at EIS testbench at GSI. Rev. Sci. Instrum. 2014, 85, 02 A956.

12. Miracoli R., Castro G., Celona L. et al. Characterization of ECR plasma by means of radial and axial X-ray diagnostics. JINST 2019, 14, C01016.

13. Mascali, D.; Castro, G.; Altana, C. et al. Electromagnetic diagnostics of ECR-Ion Sources plasmas: optical/X-ray imaging and spectroscopy. JINST 2017, 12, C12047.

14. Mascali, D.; Castro, G.; Biri, S. et al. Electron cyclotron resonance ion source plasma characterization by X-ray spectroscopy and X-ray imaging. Rev. Sci. Instrum. 2016, 87, 02A510.

15. Gumberidze, A.; Trassinelli, M.; Adrouche, N. et al. Electronic temperatures, densities, and plasma x-ray emission of a $14.5 \mathrm{GHz}$ electron-cyclotron resonance ion source. Rev. Sci. Instrum. 2010, 81, 033303.

16. Biri, S.; Valek, A.; Suta, T. Imaging of ECR plasmas with a pinhole x-ray camera. Rev. Sci. Instrum. 2004, 75, 1420.

17. Takács, E.; Radics, B.; Szabó, C.I. et al. Spatially resolved X-ray spectroscopy of an ECR plasma - indication for evaporative cooling. Nucl. Instrum. Meth. B 2005, 235, 120-125.

18. Biri, S.; Takács, E.; Rácz, R. et al. Pinhole X-Ray Camera Photographs of an ECR Ion Source Plasma. IEEE Trans. Plasma Sci. 2011, $39,11$.

19. Rácz, R.; Biri, S.; Pálinkás, J. et al. X-ray pinhole camera setups used in the Atomki ECR Laboratory for plasma diagnostics. Rev. Sci. Instrum. 2016, 87, 02A741.

20. Mascali, D.; Castro, G.; Biri, S. et al. Electron cyclotron resonance ion source plasma characterization by X-ray spectroscopy and X-ray imaging. Rev. Sci. Instrum. 2016, 87, 02A510.

21. Rácz, R.; Mascali, D.; Biri, S. et al. Electron cyclotron resonance ion source plasma characterization by energy dispersive x-ray imaging. Plasma Sources Sci. Technol. 2017, 26, 075011.

22. Romano, F. P.; Caliri, C.; Cosentino, L. et al. Macro and Micro Full Field X-Ray Fluorescence with an X-Ray Pinhole Camera Presenting High Energy and High Spatial Resolution. Anal. Chem. 2014, 86, 21.

23. Jones, B.; Deeney, C. Design of a multilayer mirror monochromatic x-ray imager for the Z accelerator. Rev. Sci. Instrum. 2004, 75, 4029.

24. McPherson, L.A.; Ampleford, D.J.; Coverdale, C.A. et al. High energy X-ray pinhole imaging at the Z facility. Rev. Sci. Instrum. 2016, 87, 063502 .

25. Bachmann, B.; Hilsabeck, T.; Fieldet, J. et al. Resolving hot spot microstructure using x-ray penumbral imaging. Rev. Sci. Instrum. 2016, 87, 11E201.

26. Sasaya, T.; Sunaguchi, N.; Hyodo, K. et al. Multi-pinhole fluorescent x-ray computed tomography for molecular imaging. Sci. Rep. 2017, 7, 5742 .

27. Biri, S.; Rácz, R.; Perduk, Z. et al. Innovative experimental setup for X-ray imaging to study energetic magnetized plasmas. JINST 2021, 16, P03003.

28. Skalyga, V.; Izotov, I.; Kalvas, T. et al. Suppression of cyclotron instability in Electron Cyclotron Resonance ion sources by two-frequency heating. Physics of Plasma 2015, 22, 083509.

29. Shalashov, A. G.; Viktorov, M.; Mansfeld, D. A. Kinetic instabilities in a mirror-confined plasma sustained by high-power microwave radiation. Physics of Plasma 2017, 24, 032111.

30. Naselli, E.; Biri, S.; Celona, L; High-resolution X-ray imaging as a powerful diagnostics tool to investigate in-plasma nuclear $\beta$-decays. Il Nuovo Cimento C 2021, 44, 64.

31. Mascali, D.; Biri, S.; Celona, L; Experimental study of single vs. two-close-frequency heating impact on confinement and losses dynamics in ECR Ion Sources plasmas by means of X-ray spectroscopy and imaging. Plasma Phys. Control. Fusion under review. 
32. Rácz, R.; Biri, S.; Perduk, Z. Effect of the two-close-frequency heating to the extracted ion beam and to the X-ray flux emitted by the ECR plasma. JINST 2018, 13, C12012.

33. Naselli, E.; Mascali, D.; Mazzaglia, M. et al. Impact of the two-close-frequency heating on ECR Ion Sources plasmas radio emission and stability. Plasma Source Science and Technology 2019, 28, 8.

34. Ryohei, T.; Koretaka, Y.; Jun, K. at al. Artificial peaks in energy dispersive X-ray spectra: sum peaks, escape peaks, and diffraction peaks. X-Ray Spectrometry 2016, 46, 5-11.

35. Kang, S.X.; Sun, X.; Ju, X. et al. Measurement and calculation of escape peak intensities in synchrotron radiation X-ray fluorescence analysis. Nuclear Instruments and Methods in Physics Research B 2002, 192, 4.

36. Karydas, A.G. and Paradellis, T. A study of silicon escape peaks for X-ray detectors with various crystal dimensions. AIP Conference Proceedings 1999, 475, 858.

37. NIST National Institute of Standards and Technology, X-Ray Form Factor, Attenuation and Scattering Tables. Available online: https:/ / physics.nist.gov/PhysRefData/FFast/html/form.html

38. Naselli, E.; Rácz, R.; Biri, S. et al. Quantitative analysis of an ECR Ar plasma structure by energy dispersive X-ray spectroscopy at high spatial resolution. JINST under review.

39. Mishra, B.; Pidatella, A.; Biri, S. et al. A novel numerical tool to study electron energy distribution functions of spatially anisotropic and non-homogeneous ECR plasmas. Physics of Plasmas 2021, 28, 102509. 